MANCHESTER

1824

군

Economics

Discussion Paper Series EDP-1009

\title{
Dynamic relational contracts with credit constraints
}

\author{
Jonathan Thomas \\ Tim Worrall
}

April 2010

Economics

School of Social Sciences

The University of Manchester

Manchester M13 9PL 
University of Manchester · Economics Discussion Paper Series · EDP1009 · April 2010

\title{
DYNAMIC RELATIONAL CONTRACTS WITH CREDIT CONSTRAINTS*
}

\author{
Jonathan P. Thomas and Tim WorralL**
}

This paper considers a long-term relationship between two agents who undertake costly actions or investments which produce a joint benefit. Agents have an opportunity to expropriate some of the joint benefit for their own use. The question asked is how to structure the investments and division of the surplus over time so as to avoid expropriation. It is shown that investments may be either above or below the efficient level and that actions and the division of the surplus converges to a stationary solution at which either both investment levels are efficient or both are below the efficient level.

KEYWORDS: Credit constraints, Limited commitment, Relational contracts, Selfenforcing contracts.

JEL Classification: C61, C73, D86, D91, L14.

\section{INTRODUCTION}

It is often difficult to enforce contracts. This may be because the terms of the contracts are difficult to specify precisely or because they are difficult to specify in a way verifiable to a court. It may be that there is no legal authority to enforce a contract. When relationships are repeated it is possible to include an element of self-enforcement in the contract by designing terms so that any short-term incentive to renege is offset by a long-term benefit from adhering to the contract. Non-stationary contracts can do better in this regard as the future benefits from the contract can be tailored to the current situation, favoring the agent who has most temptation to renege in the current period.

We consider such a self-enforcing or relational contract in the case where two risk-neutral agents make repeated costly relation-specific investments or actions $a_{i}$ that produce a joint output $y\left(a_{1}, a_{2}, s\right)$ to be shared at each date (where $s$ denotes the current state of nature). Agents observe actions and output and have a common discount rate. Initially we assume that agents can neither borrow nor save so that current consumption depends only on the division of current output although later we maintain the borrowing constraint but allow agents to save. Contracts cannot be enforced and in the event of disagreement

School of Economics, The University of Edinburgh, 31 Buccleuch Place, Edinburgh, EH8 9JT, U.K., email: jonathan.thomas@edinburgh.ac.uk, and Economics, School of Social Sciences, University of Manchester, Oxford Road, Manchester, M13 9PL, U.K., email: tim. worrall@manchester.ac.uk.

${ }^{*}$ We gratefully acknowledge the financial support of the Economic and Social Research Council (Research Grant: RES-000-23-0865). We also thank in particular Pierre Dubois, Francisco Gonzalez, Bruno Jullien and John Moore for helpful comments. An earlier version of this paper had the title "Self-enforcing contracts with action dynamics". All errors are ours.

${ }^{* *}$ The second author gratefully acknowledges the support of the Hallsworth Research Fellowship Fund at the University of Manchester. 
agent $i$ receives a current gross breakdown payoff of $\phi_{i}\left(a_{1}, a_{2}, s\right) .{ }^{1}$ We assume that the breakdown payoff of agent $i$ is increasing in the investment of agent $j$ (whenever $j$ has a productive investment). In this case if say, agent 1's investment is increased, more must be offered to agent 2 to prevent him reneging. Thus although the joint surplus may be increased, agent 1 may have no incentive to increase her investment or action as there may be no division of the surplus which simultaneously prevents agent 2 from reneging and compensates agent 1 for the increased investment: agents will face a hold-up problem and investment may be inefficiently low.

One-sided-action versions of this model or variations on it, have been recently studied in a growing literature (see, e.g., Albuquerque and Hopenhayn, 2004; Kovrijnykh, 2009; Sigouin, 2003; Thomas and Worrall, 1994). This literature has for the most part considered the case where the side taking the action can commit to a greater or lesser extent, ${ }^{2}$ and the action is assumed to be observable to the agents in the relationship (but see Footnote 3 for exceptions to the latter). Results have shown how payments to one agent may be backloaded and consequently allow the action or investment of the other agent to be increased in the future thus generating dynamics in the agents's actions as well as in monetary payments. This approach has been usefully applied in a number of different contexts (such as international capital flows, firm dynamics, borrowing and lending, international trade and formal and informal sectors etc.). It has been extended in a number of directions, for example, Cooley et al. (2004) embed a model of this type in a general equilibrium context to make the default option endogenous; Hopenhayn and Werning (2008) consider unobservable outside options; Atkeson and Cole (2008) allow for private information about output within each period; Özgür (2004) allows for lumpy investment; Popov (2008) considers a costly enforcement technology; Hauser and Uysal (2006) consider one-sided provision of a public good; Quintin (2008) has a two sector model with formal and informal sectors and Board (2008) considers the case of multiple agents where the principal, the party taking the action that is subject to hold-up, can trade with only one agent in a period. (Other references are discussed below and in Section 6.)

There is also a broader literature on relational contracts (see, e.g., Doornik, 2006; Levin, 2003; Rayo, 2007) which builds upon the work of Macleod and Malcomson (1989), has studied models with more general ingredients (including two- or many-sided actions, enforceable payments, and also such features as moral hazard, hidden information, and endogenous property rights), but has restricted attention to stationary equilibria, either because they are optimal due to assumed unlimited liability, or because the focus is on organizational structures under which full efficiency can be achieved, thus eliminating any interesting dynamics

\footnotetext{
${ }^{1}$ The breakdown payoffs are assumed to be feasible, $\phi_{1}\left(a_{1}, a_{2}, s\right)+\phi_{2}\left(a_{1}, a_{2}, s\right) \leq y\left(a_{1}, a_{2}, s\right)$. Details of the model will be specified in Section 2 and discussed further in Section 6 .

${ }^{2}$ Typically, if agent 1 is the party undertaking a repeated investment, then $\phi_{2}\left(a_{1}, s\right)=y\left(a_{1}, s\right)$, that is, agent 2 (who cannot commit) is able to expropriate current output.
} 
in investments. ${ }^{3}$ Our paper bridges these two literatures in examining the two sided action case (although our model also encompasses the one-sided action case when there is limited commitment on both sides). ${ }^{4}$ As in the one-sided action models we impose limited liability or borrowing constraints. As in much of the relational contracting literature we allow for both sides to the contract to undertake repeated investments.

We establish three main results. Firstly we derive a backloading result (Theorem 1). In general it is well known that optimum self-enforcing contracts are often subject to the backloading principle. ${ }^{5}$ This is easiest to see in the case where both agents are risk-neutral and discount the future at the same rate, and one agent is able to commit to the contract. Then the backloading principle says that transfers to the agent who cannot commit should be backloaded into the future. ${ }^{6}$ The intuition is the following. Suppose that of the two agents, agent 1 can commit to the contract but agent 2 cannot. Further, suppose that agent 1 has most of the

\footnotetext{
${ }^{3}$ Some recent work has considered the implications of introducing a limited liability constraint into a moral hazard model with self-enforcement constraints. Fong and Li (2009) do this for the model of Levin (2003). The characterization of equilibria changes substantially. They show that if the principal extracts most of the surplus, the backloading of the agent's utility can lead to a probationary contract in which the agent's consumption is initially at the lower bound, and incentives are provided by the threat of termination; at some point this threat is removed and pay increases to a higher level. Likewise, Yang (2009) assumes limited liability in a model with both moral hazard and adverse selection, and shows that backloading occurs, although adverse selection provides an additional reason for backloading pay.

${ }^{4}$ There is a literature on risk-sharing and two-sided limited commitment and no actions (which are subject to hold-up) in which the optimum contract evolves toward a stationary but typically non-degenerate distribution of future expected utilities (see, e.g., Kocherlakota, 1996; Ligon et al., 2002; Thomas and Worrall, 1988). For this result the two-sided lack of commitment is crucial as if there is only one-sided lack of commitment utilities will also converge to a degenerate distribution. This is in contrast to the results of the current paper where convergence occurs even in the two-sided case. Moreover, we show that if efficiency cannot be sustained in the stationary phase then both agents are simultaneously constrained and this feature again makes our results qualitatively distinct from the risk-sharing models with no actions where in any non-degenerate contract at most one agent's constraint will bind at a time.

${ }^{5}$ Ray (2002) has established the most general backloading result. He considers a very general (non-stochastic) principal-agent model in which both parties can potentially take actions, but the principal can commit within each period, so the self-enforcement constraint only applies to the agent. He shows that an efficient contract has terms that move in favor of the agent, converging in finite time to the efficient self-enforcing continuation that maximizes the agent's payoff. Strikingly, this is true even with non-transferable utility. In our two-sided limited commitment case we have an analogous result with convergence to a continuation which maximizes the sum of payoffs across the two agents. (In fact, in the transferable utility case that we consider, maximizing the agent's payoff in the one-sided case also maximizes the sum of payoffs.)

${ }^{6}$ If the agent who cannot commit has a lower discount factor than the other agent then this can work against backloading (see Aguiar and Amador, 2009; Aguiar et al., 2009; Opp, 2010). Acemoglu et al. (2008) consider a model in which there are atomistic agents who invest/supply labor each period, and a government which can expropriate output; nevertheless it shares a similar structure with the one sided models discussed above- the government which expropriates more than it should is punished by not being reelected. They show that backloading and convergence, as in Ray (2002), may not occur depending on the discount factor asymmetry, but that risk-aversion and capital accumulation also play a role in this result.
} 
ex ante bargaining power, so that agent 2 is getting a relatively low discounted utility from the contract. This may impose an efficiency cost on the contract if the investment of agent 1 needs to be kept low to limit the gains to agent 2 from expropriation, who has little to lose. Since both agents are risk-neutral they are concerned only with the discounted value of utility (transfers net of action costs) and not the actual timing of utility received. Thus backloading transfers to agent 2 as much as possible, whilst keeping the discounted sum of transfers unchanged, costs nothing, but provides a carrot in the future which would be forgone if agent 2 reneged. Such a change doesn't worsen current incentives but improves future incentives by increasing agent 2's continuation utilities - they rise as the backloaded payments phase is approached - and hence this allows future increases in agent 1's investment. ${ }^{7}$ In our context where both agents make investments and neither agent can commit, the operation of this principle is less clear. For example, agent 1 herself may be constrained later, so this backloading could worsen future incentives. We show, however, that the backloading principle remains partially valid and that we have backloading of consumption for at most one of the agents (whose identity depends on the initial split in the surplus from the relationship).

Furthermore it is shown that as the backloading principle applies to utilities and not simply consumption it might be optimal to increase investment beyond the efficient level. This allows more output to be allocated to the other agent and thus more backloading. Of course there is an efficiency loss in overinvesting so it will always be desirable to backload transfers as much as possible before backloading utility by altering actions. The result however, has the implication that an optimum self-enforcing contract may involve overinvestment in the initial periods by one of the agents, despite the hold-up problem suggesting that there will be underinvestment. Nevertheless we shall show that it will never be the case that both agents overinvest - even at different dates - in any equilibrium. Equally we are able to show that in the case where only one agent takes an action (as in the literature mentioned above) there is never overinvestment (Theorem 4).

Secondly, we establish that the contract converges to a stationary phase in finite time with probability one (Theorem 2). It is shown that this stationary phase corresponds to the self-enforcing contract which maximizes the current net surplus and hence the joint utility of the two agents. ${ }^{8}$ Our result is general: it holds even when the default payoffs and production technologies fluctuate through time and our stochastic structure is general enough to encompass cases where the action choices are made sequentially at alternative periods rather than simultaneously. Furthermore we show that in the stationary phase for a given state either both agents are investing efficiently (which happens if this is feasible

\footnotetext{
${ }^{7}$ A limited liability constraint, typically imposed as no borrowing, is needed to prevent the backloading taking an extreme form in which agent 2 receives a large negative payment in the first period which would allow efficiency to be attained from the second period.

${ }^{8}$ In fact we also establish the converse that any self-enforcing contract which maximizes the joint utility of the two agents also maximizes the current surplus at each date.
} 
given the self-enforcement constraints) or both are underinvesting. Likewise in this stationary state, unless the first best is attainable, we show that both agents are simultaneously constrained in the sense that they are both at the point of reneging on the contract and taking their default payoffs.

Thirdly we show that if the optimum contract is non-trivial with positive investment by both agents at each date then it will exhibit a two-phase property (Theorem 3). ${ }^{9}$ In the first phase there is backloading with zero consumption and overinvestment by one of the agents. Although this first phase may not exist depending on the initial split of overall surplus and discount factor we present an example in which it does. In the second phase (which occurs with probability one) there will be no overinvestment. In this second phase, there can be first a one-period transition in which one of the agents is investing efficiently, and thereafter either both actions are efficient, or both actions are inefficient and both agents are indifferent to reneging on the contract. The subsequent part of this phase is stationary and joint utility maximizing as explained above.

Finally we show (Theorem 5) that the if the interest rate at which agents can save is no greater than the discount rate then the set of efficient self-enforcing contracts is independent of the interest rate. In principle it might be possible to use savings to relax the incentive constraints. For example, if agent 1 saves some of her early transfers and then makes a transfer out of savings to agent 2 at a later date, the increase in the payoff to agent 2 may, if the transfer is credible, allow agent 1 to increase her action towards the efficient level. However, as the backloading result suggests, this will not be desirable because in the backloading phase only the unconstrained agent can save so that any subsequent transfer is likely to lead to default or not improve the other agent's continuation payoff.

Although this paper significantly extends existing results it does so by adopting a different method. The literature cited above typically uses a dynamic programming approach to characterize optimum self-enforcing contracts. In our context the dynamic programming approach has the disadvantage that the resulting problem may be non-convex and, even when it is convex, it is known (see, e.g., Thomas and Worrall, 1994) that the value function may not be differentiable. Thus the use of first-order conditions is typically problematical. ${ }^{10}$ We avoid these issues by deriving results from more straightforward variational methods and our main results will not require that the set of constrained efficient contracts be

\footnotetext{
${ }^{9}$ Again, this is reminiscent of the result established by Ray (2002).

${ }^{10}$ If both agents are investing, the value function will be differentiable if it is concave. If only one agent is investing the value function will not be differentiable in general even if it is concave. These points of non-differentiability can also be an important part of the solution so that even with concavity a sub-differential analysis must be used. This is in contrast to the dynamic moral hazard problem analyzed by Pavoni (2009) who is able use a first-order approach despite points of non-differentiability by showing that such points are almost never reached at the optimum.
} 
convex. ${ }^{11}$ This allows us to derive our results in more generality whilst avoiding some of the technical requirements of the dynamic programming approach.

The paper proceeds as follows. The next section describes the model and optimum self-enforcing contracts. Section 3 provides the main results of the paper. Section 4 considers the special case where only one agent contributes to production. Section 5 allows for agents to save subject to borrowing constraints. Section 6 discusses how our results generalize to different assumptions about the breakdown and punishment payoffs and Section 7 concludes.

\section{MODEL}

We consider a dynamic model of joint production where agents repeatedly undertake some action or investment that generates a joint output. Agents can agree on the actions to undertake and how to divide up the output but can also default on any agreement and receive an alternative breakdown payoff. In this section we shall describe the economic environment, the joint production and action sets, the breakdown payoffs and the set of self-enforcing contracts. In addition we shall define a game played by the two agents and identify selfenforcing contracts with the subgame perfect equilibria of that game. Our interest will be in optimal self-enforcing contracts or equivalently the Pareto-efficient subgame perfect equilibria.

\subsection{Economic environment}

Time is discrete and indexed by $t=0,1,2, \ldots, \infty$. The environment is uncertain and at the start of each date a state of nature $s$ is realized from a finite state space $\mathcal{S}=\left\{s_{1}, s_{2}, \ldots, s_{n}\right\}$. The state evolves according to a time homogeneous Markov process with transition matrix $\left[\pi_{s r}\right]$ with $\sum_{r \in \mathcal{S}} \pi_{s r}=1$, all $s \in \mathcal{S}$, and we assume some initial state $s_{0}$ has probability one. We shall assume that the Markov chain is irreducible so that every state communicates with every other. ${ }^{12}$ This structure is general and encompasses the case with no uncertainty where $n=1$ and the possibility that some $\pi_{s r}=0$. We shall denote the state at date $t$ by $s_{t}$ and the history of states will be denoted $s^{t}=\left\{s_{0}, s_{1}, s_{2}, \ldots, s_{t}\right\} .{ }^{13}$

\footnotetext{
${ }^{11}$ Although it would be possible to convexify the problem by allowing for random contracts, we prefer to concentrate on pure strategy equilibria, partly because our results show that even in this case strong convergence results can be established.

${ }^{12} \mathrm{~A}$ number of these assumptions are inessential and made for convenience and simplicity. The important property is that the stochastic process is Markovian. Irreducibility is made for convenience as it is used for the later convergence results. Equally we could we specify a distribution over the set of initial states rather than assuming there is a fixed initial state. For many results it is possible to assume that there is some finite time horizon $T$ although we shall be interested in convergence properties of optimum contracts and these results will require an infinite time horizon.

${ }^{13}$ Where we write $s^{t}$ we shall assume this is a positive probability event unless otherwise stated, as zero probability events play no role here.
} 
There are two agents, $i=1,2$, and at each date both agents choose an action or investment $a_{i}$ from $\Re_{+}{ }^{14}$ Actions are taken simultaneously after the state is realized. Actions lead to an output $y\left(a, s_{t}\right)$, where $a \equiv\left(a_{1}, a_{2}\right)$, that may depend upon the current state. Output, actions and states are observed by both agents.

In Section 5 we shall allow agents to save subject to borrowing constraints in which case the division of the surplus need not correspond with the consumption. However, we shall show in that section that provided the interest rate is below the discount rate savings will not affect the set of efficient contracts. Thus it is useful to start with the assumption that each agent's consumption does correspond to their division of the joint output. Exactly how this division is determined is described in the next sub-section. For the moment we shall simply think of a consumption and action plan for each agent that depends on the history $s^{t}$. Denote the consumption of agent $i$ at history $s^{t}$ by $c_{i}\left(s^{t}\right)$ and the action by $a_{i}\left(s^{t}\right)$. Consumption is assumed non-negative so the feasible set of consumptions at time $t$ in state $s$ satisfies $c_{i}\left(s^{t}\right) \geq 0$, for $i=1,2$ and $c_{1}\left(s^{t}\right)+c_{2}\left(s^{t}\right) \leq y\left(a\left(s^{t}\right), s_{t}\right)$ for all $s^{t}$.

We assume that agents have time separable utility functions, are risk neutral and that action costs are linear: ${ }^{15}$ agent $i$ 's utility at $t$ is given by

$$
w_{i}\left(s^{t}\right)=c_{i}\left(s^{t}\right)-a_{i}\left(s^{t}\right) .
$$

We assume that both agents discount the future by a common factor $\delta \in(0,1)$ and that agents are interested in maximizing expected discounted utility

$$
\mathrm{E}\left[\sum_{t=0}^{\infty} \delta^{t} w_{i}\left(s^{t}\right) \mid s_{0}\right] .
$$

For a given pair of actions the current net surplus generated at history $s^{t}$ is $y\left(a\left(s^{t}\right), s_{t}\right)-\left(a_{1}\left(s^{t}\right)+a_{2}\left(s^{t}\right)\right)$. The feasible set of per-period utility payoffs satisfies $w_{i}\left(s^{t}\right) \geq-a_{i}\left(s^{t}\right)$ for $i=1,2$ and $\sum_{i} w_{i}\left(s^{t}\right)+a_{i}\left(s^{t}\right) \leq y\left(a\left(s^{t}\right), s_{t}\right)$ for all $s^{t}$.

We make the following assumption about the production function.

Assumption 1 The function $y\left(\cdot, \cdot, s_{t}\right): \Re_{+}^{2} \rightarrow \Re_{+}$satisfies the following conditions: (i) Output at zero: $y(0,0 ; \cdot)=0$; (ii) Continuity: it is continuous in $a$ on $\Re_{+}^{2}$; (iii) Differentiability: it is twice continuously differentiable in $a$ on $\Re_{++}^{2}$; (iv) Monotonicity: either there exists $i \in\{1,2\}$ such that it is constant in $a_{i}$ for

\footnotetext{
${ }^{14}$ We shall use the notation $\Re_{+}$to denote the non-negative orthants of the real numbers and $\Re_{++}$to denote its interior.

${ }^{15} \mathrm{As}$ is fairly standard this linearity assumption is made for convenience and the analysis will carry through if actions costs are convex. Thus suppose $w_{i}=c_{i}-g_{i}\left(a_{i}\right)$ where $g_{i}$ is strictly increasing and convex and $g(0)=0$. Letting $h_{i}$ denote the inverse of $g_{i}$ we have $a_{i}=h_{i}\left(g_{i}\right)$ where $h_{i}$ is strictly increasing and concave. Hence agents can be viewed as choosing $g_{i}$ and the reduced-form production function is $f\left(g_{1}, g_{2}, s_{t}\right)=y\left(h_{1}\left(g_{1}\right), h_{2}\left(g_{2}\right), s_{t}\right)$ which will satisfy Assumption 1 (below) on the production function with $g_{i}$ replacing $a_{i}$ and $f$ replacing $y$. In this case the net surplus is $f\left(g_{1}, g_{2}, s_{t}\right)-g_{1}-g_{2}$.
} 
all $a_{j} \geq 0$ and strictly increasing in $a_{j}, j \neq i$ or strictly increasing in $\left(a_{1}, a_{2}\right)$ on $\Re_{++}^{2}$; (v) Diminishing marginal product: $\partial^{2} y / \partial a_{i}^{2}<0$ whenever $\partial y / \partial a_{i}>0$; (vi) Boundedness: $\lim _{\alpha \rightarrow \infty} y\left(\alpha a_{1}, \alpha a_{2}, s_{t}\right) / \alpha<a_{1}+a_{2}$ for all $a \in \Re_{++}^{2}$;

These are standard assumptions on the production function. The monotonicity assumption we make allows us to cover the case where only one action matters for production. In this situation although we formally allow both agents to choose an action, this will be equivalent, in an efficient equilibrium, to imposing the restriction that the action is zero. We impose Assumption 1(vi) so that in conjunction with the other conditions the set of action choices that yield nonnegative surplus, $\tilde{A}\left(s_{t}\right)=\left\{\left(a_{1}, a_{2}\right) \in R_{+}^{2} \mid y\left(a_{1}, a_{2}, s_{t}\right) \geq a_{1}+a_{2}\right\}$, is compact. Since all assumptions apply state-wise we shall often be able to drop the notational dependence on the state where this is convenient.

REMARK The production technology and stochastic structure is sufficiently general to encompass a situation where in some states only agent 1 takes an action and in other states only agent 2 takes an action. For example, one agent takes an action in even periods and the other agent takes an action in odd periods. With some minor modifications of the proofs our monotonicity assumption can easily be relaxed to cover the case where in some states neither agent takes an action and output is always zero.

Since actions are chosen simultaneously and independently we define the conditionally efficient actions $a_{i}^{*}\left(a_{j}, s_{t}\right)$ such that

$$
a_{i}^{*}\left(a_{j}, s_{t}\right) \in \underset{a_{i} \in \Re_{+}}{\arg \max }\left[y\left(a_{1}, a_{2}, s_{t}\right)-a_{i}\right] .
$$

We then have the following standard result. ${ }^{16}$

Lemma 1 Given Assumption 1 the conditionally efficient actions are singlevalued, continuous functions of the other agent's action.

We define an efficient action pair $a^{* *}\left(s_{t}\right)=\left(a_{1}^{* *}\left(s_{t}\right), a_{2}^{* *}\left(s_{t}\right)\right)$ where $a_{i}^{* *}\left(s_{t}\right)=$ $a_{i}^{*}\left(a_{j}^{* *}\left(s_{t}\right), s_{t}\right)$ for $i \neq j=1,2$ and assume ${ }^{17}$

Assumption 2 The efficient action pair $a^{* *}\left(s_{t}\right)$ is unique for each $s_{t}$.

As we have not imposed a profitability condition that there exists a vector $a$ such that $y\left(a_{1}, a_{2}, s_{t}\right)>a_{1}+a_{2}$ it may be that $a^{* *}\left(s_{t}\right)=(0,0)$ in some state or indeed in all states. ${ }^{18}$

\footnotetext{
${ }^{16}$ All proofs are given in the Appendix.

${ }^{17}$ It would be possible to derive this assumption by additionally assuming that the function $y\left(a_{1}, a_{2}, \cdot\right)$ is strictly concave if it is increasing in both its arguments. However, we do not require this strict concavity elsewhere, so it is simpler to directly assume that the efficient action pair is unique.

${ }^{18}$ Our results will apply (trivially) in this case.
} 


\subsection{The breakdown game}

In this and the following sections we specify how agents agree on the division of the surplus and what happens in the event of disagreement. In each period agents must decide how to act and how to divide up the subsequent surplus. We shall suppose that a period is split into two stages with actions being determined at the first stage and the division of the surplus/recourse to breakdown taking place at the second stage after output is known (recall that uncertainty is resolved before the action decision).

We start by defining breakdown payoffs. These represent what an agent can guarantee herself given the actions taken by both agents, and are given by

$$
\phi_{i}\left(a_{1}, a_{2}, s_{t}\right)-a_{i}
$$

for agent $i$ in state $s_{t}$. They may reflect the property rights of the two agents over output, for example, specifying a fixed percentage split. An agent can always take the option of receiving her breakdown payoff. Analogous to Assumption 1 we shall assume that the breakdown payoffs satisfy:

Assumption 3 The function $\phi_{i}\left(\cdot, \cdot, s_{t}\right): \Re_{+}^{2} \rightarrow \Re_{+}$is twice continuously differentiable on $\Re_{++}^{2}$, with $\partial \phi_{i}\left(a, s_{t}\right) / \partial a_{i} \geq 0$. Whenever $\partial \phi_{i}\left(a, s_{t}\right) / \partial a_{i}>0$ then $\partial^{2} \phi_{i}\left(a, s_{t}\right) / \partial a_{i}^{2}<0$. Moreover $\partial^{2} \phi_{i}\left(a_{1}, a_{2}\right) / \partial a_{1} \partial a_{2} \geq 0$ (complementarity). In addition, the $\phi_{i}$ are feasible, i.e., $\phi_{1}\left(a, s_{t}\right)+\phi_{2}\left(a, s_{t}\right) \leq y\left(a, s_{t}\right)$ for each $a$ and $s^{t}$; $\partial y\left(a, s_{t}\right) / \partial a_{i}>0$ implies $\partial \phi_{j}\left(a, s_{t}\right) / \partial a_{i}>0, j \neq i$ (hold-up); and

$$
\frac{\partial \phi_{1}\left(a, s_{t}\right)}{\partial a_{i}}+\frac{\partial \phi_{2}\left(a, s_{t}\right)}{\partial a_{i}} \leq \frac{\partial y\left(a, s_{t}\right)}{\partial a_{i}} \quad \forall s_{t} \text { and } i=1,2 .
$$

The assumption of complementarity is general enough to cover almost all cases studied in the existing literature, while implying that the reaction functions in the breakdown game are (weakly) upward sloping, something that simplifies the arguments below. Equation (1) requires that the increase in the total breakdown payoff cannot exceed the marginal product. If it is assumed that $\phi_{i}(0,0, \cdot)=0$ for $i=1,2$, then equation (1) together with $y(0,0, \cdot)=0$ (from Assumption 1) implies that the breakdown payoffs are feasible, $\phi_{1}\left(a, s_{t}\right)+\phi_{2}\left(a, s_{t}\right) \leq y\left(a, s_{t}\right)$.

REMARK Assuming $\partial y\left(a_{1}, a_{2}, s_{t}\right) / \partial a_{i}>0$ implies that $\partial \phi_{j}\left(a_{1}, a_{2}, s_{t}\right) / \partial a_{i}>0$ reflects our hold-up assumption. It is made to avoid the case where agent $i$ 's contribution to output does not increase agent $j$ 's claim on output. In such a case hold-up and underinvestment by agent $j$ cannot occur in any efficient equilibrium. Situations with hold-up are our primary focus, and the assumption allows us to streamline the arguments below.

Assumption 3 places relatively few restrictions on the breakdown payoffs. They must be feasible and satisfy equation (1) but we do not require that 
they exhaust available output. For example, disagreement may incur a cost, such as lawyers' fees, which leads to a loss of output. We mention two special cases which satisfy Assumption 3. First, we may have that each agent can extract a fraction (possibly state-dependent) of output in the breakdown. In this case $\phi_{i}\left(a_{1}, a_{2}, s_{t}\right)=\theta_{i}\left(s_{t}\right) y\left(a_{1}, a_{2}, s_{t}\right)$ and Assumption 3 is satisfied provided $\theta_{i}\left(s_{t}\right)>0, i=1,2$, and $\sum_{i=1}^{2} \theta_{i}\left(s_{t}\right) \leq 1$. Secondly, suppose that output is an additive function of inputs such that $y\left(a_{1}, a_{2}, s_{t}\right)=f_{1}\left(a_{1}, s_{t}\right)+f_{2}\left(a_{2}, s_{t}\right)$ and $\phi_{i}\left(a_{1}, a_{2}, s_{t}\right)=\theta_{i 1}\left(s_{t}\right) f_{1}\left(a_{1}, s_{t}\right)+\theta_{i 2}\left(s_{t}\right) f_{2}\left(a_{2}, s_{t}\right)$. In this case agents make separate contributions to joint output and can capture some of their own and some of the other agent's contribution in the breakdown. ${ }^{19}$ Then Assumption 3 is satisfied provided $\theta_{i j}\left(s_{t}\right) \geq 0$ with this being strict for $i \neq j$, and $\sum_{i=1}^{2} \theta_{i j}\left(s_{t}\right) \leq$ $1, j=1,2$. This latter additive structure includes the case which has been predominantly studied in the literature where only the action of one agent is productive and the other can extract the entire output in the breakdown; for example, $f_{2}=0$ and $\theta_{21}=1$ and this case is considered in Section 4.

An important part of the analysis will be related to the best response in the breakdown game. Denote the best-response functions (functions because of the strict concavity the function $\phi_{i}\left(a_{1}, a_{2}, \cdot\right)$ in its own argument made in Assumption 3) as

$$
a_{i}^{N}\left(a_{j}, s_{t}\right)=\underset{\tilde{a}_{i} \in \Re_{+}}{\arg \max }\left\{\phi_{i}\left(\tilde{a}_{i}, a_{j}, s_{t}\right)-\tilde{a}_{i}\right\} .
$$

Lemma 2 Given Assumption $3, a_{i}^{N}\left(a_{j}, s_{t}\right)$ is continuous and weakly increasing in $a_{j}$. Moreover we have $a_{i}^{N}\left(a_{j}, s_{t}\right) \leq a_{i}^{*}\left(a_{j}, s_{t}\right)$ for each $a_{j}$ and every state $s_{t}$ with strict inequality whenever $a_{i}^{*}\left(a_{j}, s_{t}\right)>0$.

A Nash equilibrium of the breakdown game occurs where the best-response functions intersect (existence follows by standard arguments). As with the efficient action pair $a_{i}^{* *}\left(s_{t}\right)$, without further assumptions the Nash equilibrium need not be unique. However, the potential non-uniqueness is not critical as the Nash equilibria can be Pareto-ranked (as the best-response functions are nondecreasing). Henceforth we let $\left(a_{1}^{N E}\left(s_{t}\right), a_{2}^{N E}\left(s_{t}\right)\right)$ denote the dominant Nash equilibrium and all our results apply relative to this dominant Nash equilibrium. ${ }^{20}$

\subsection{Equilibria}

Here, we formulate equilibria as action and consumption plans (or equivalently action and utility plans) which are immune to deviations in which an agent takes her breakdown payoff.

Given that we allow for current surplus to exceed the sum of breakdown payoffs, it is necessary to specify a mechanism for splitting the surplus at the

\footnotetext{
${ }^{19}$ This proportional formulation has also been studied in the relational contracting literature (e.g., Halonen, 2002).

${ }^{20}$ See Section 6 for a discussion of this assumption.
} 
division stage of the game. We suppose for simplicity that the agents play a Nash demand game. ${ }^{21}$ In this Nash demand game both agents simultaneously announce utility claims $\left(w_{1}, w_{2}\right)$. If these claims are feasible, as defined above, then this determines the split of the surplus. ${ }^{22}$ If they are not feasible, then agents receive their breakdown payoffs.

To specify what happens in the dynamic game played by the agents we shall consider punishments where the breakdown game is triggered as soon as there is any deviation, and thereafter play remains there (i.e., the breakdown game is triggered in every subsequent period). Although the other agent cannot change her action immediately in response to the deviation, the breakdown is triggered in the period of deviation to determine payoffs in that period - the action of the non-deviator will still be at the equilibrium level, and from the next period the Nash equilibrium of the breakdown game is played. We compute equilibria relative to these punishments. Specifically, suppose that $a$ is the current recommended action vector. If agent $i$ is to deviate then the best the agent can do is to choose the best response action $a_{i}^{N}\left(a_{j}, s_{t}\right) \cdot{ }^{23}$ She will be punished thereafter by Nash reversion in which both agents choose their best responses and hence both will play the (dominant) Nash equilibrium of the breakdown game. ${ }^{24}$ Write $D_{i}\left(a_{j}, s_{t}\right)$ to denote the corresponding best non-cooperative discounted payoff that agent $i$ can get starting from agent $j$ 's action $a_{j}$ in state $s_{t}$. We refer to $D_{i}\left(a_{j}, s_{t}\right)$ as the deviation payoff. It is defined recursively as

$$
D_{i}\left(a_{j}, s_{t}\right)=\phi_{i}\left(a_{i}^{N}\left(a_{j}, s_{t}\right), a_{j}, s_{t}\right)-a_{i}^{N}\left(a_{j}, s_{t}\right)+\delta \sum_{s_{t+1} \in \mathcal{S}} \pi_{s_{t} s_{t+1}} D_{i}\left(a_{j}^{N E}\left(s_{t+1}\right), s_{t+1}\right)
$$

where $D_{i}\left(a_{j}^{N E}\left(s_{t+1}\right), s_{t+1}\right)$ is the deviation continuation payoff from the play of the Nash equilibrium. Given our hold-up assumption (see Assumption 3) it follows that the deviation payoff is strictly increasing in the action of the other agent

\footnotetext{
${ }^{21}$ What we want to capture is that there is an ex ante agreement on what actions should be taken and how the resulting output should be split, and that failure to abide by it will lead to the breakdown payoffs. The Nash demand game is a simple way of implementing this idea, but we stress that our results are not sensitive to the way it is operationalized. See Section 6 for further discussion and how this relates to the existing literature.

${ }^{22}$ We shall henceforth ignore equilibria such that less than available output is claimed, as these are Pareto-dominated and our interest will be in Pareto-efficient equilibria. (Alternatively we could specify that breakdown is triggered whenever the sum of demands is unequal to output; the efficient equilibria are the same in either case.)

${ }^{23}$ We need only consider deviations at the choice of action stage since if an agent were to contemplate deviation at the surplus division stage, the breakdown payoff would be the same except that her action would not be optimized to maximize the breakdown payoff. (Recall we are restricting attention to equilibria where all output is claimed in the demand game, and given that only an increased demand could be profitable, this must trigger the breakdown game.) Thus an agent can do no worse than deviate at the action choice stage, choosing the best-reply action.

${ }^{24}$ Punishment by immediate triggering of the breakdown game, followed by repeated play of the (dominant) Nash equilibrium of the breakdown game is a subgame perfect equilibrium of the overall game (each agent just demands the whole output from the point of deviation on); see the proof of Lemma 4 .
} 
when the other agent's action increases output. This and other properties of the deviation payoff are stated in the following lemma.

Lemma 3 The deviation payoff $D_{i}\left(a_{j}, s_{t}\right)$ is a continuous, non-decreasing function of $a_{j}$; it is differentiable for $a_{i}^{N}\left(a_{j}\right)>0$ and $D_{i}\left(a_{j}, s_{t}\right) \geq 0 . D_{i}\left(a_{j}, s_{t}\right)$ is strictly increasing in $a_{j}$ whenever $\partial y\left(a_{i}^{N}\left(a_{j}\right), a_{j}\right) / \partial a_{j}>0$.

We consider pure-strategy subgame perfect equilibria of the above game. Let the putative outcome path of an equilibrium be represented by $\left\{a\left(s^{t}\right), w\left(s^{t}\right)\right\}_{t=0}^{\infty}$, where $a\left(s^{t}\right)$ and $w\left(s^{t}\right)$ are the respective actions and actual payoff divisions (not demands) at time $t$ along the equilibrium path. This includes the possibility that breakdown has occurred in any period, in which case $w\left(s^{t}\right)=\phi\left(a\left(s^{t}\right), s_{t}\right)-$ $a\left(s^{t}\right)$. The outcome path $\left\{a\left(s^{t}\right), w\left(s^{t}\right)\right\}_{t=0}^{\infty}$ is feasible if $w\left(s^{t}\right)+a\left(s^{t}\right) \geq 0$ and $\sum_{i} w_{i}\left(s^{t}\right)+a_{i}\left(s^{t}\right) \leq y\left(a\left(s^{t}\right), s_{t}\right)$ for every history $s^{t}$.

As has been stated we assume that there is Nash-reversion after any deviation and compute equilibria relative to these deviation payoffs. Then necessary and sufficient conditions for this path to be equilibrium relative to Nash-reversion is that it is feasible, and that for every $s^{t}$ and $i=1,2$,

$$
V_{i}\left(s^{t}\right) \equiv w_{i}\left(s^{t}\right)+\mathrm{E}\left[\sum_{\tau=t+1}^{\infty} \delta^{\tau-t} w_{i}\left(s^{\tau}\right) \mid s^{t}\right] \geq D_{i}\left(a_{j}\left(s^{t}\right), s_{t}\right) .
$$

The payoff $V_{i}\left(s^{t}\right)$ is the discounted payoff to $t$ that agent $i$ anticipates from the equilibrium, while the right hand side of (2) is the deviation payoff she would get from deviating from the recommended action $a\left(s^{t}\right)$ after the history $s^{t}$. We shall refer to the $V_{i}\left(s^{t}\right)$ as the continuation utilities. Whenever (2) holds with equality we say that agent $i$ is constrained - any reduction in her on-equilibrium path payoff would lead her to deviate at $s^{t}$; otherwise we say that agent $i$ is unconstrained. We refer to the inequalities (2) as the self-enforcing or incentive constraints.

The following is standard. A contract or agreement $\Gamma$, specifies history contingent actions and utilities $a\left(s^{t}\right)$ and $w\left(s^{t}\right)$ at each date in each state, $\Gamma=$ $\left\{a\left(s^{t}\right), w\left(s^{t}\right)\right\}_{t=0}^{\infty}$. It is self-enforcing if it is both feasible and satisfies the selfenforcing constraints. A self-enforcing agreement then corresponds to a pure strategy subgame perfect equilibrium of the game. We shall denote the restriction of a contract after the history $s^{t}$ by $\Gamma\left(s^{t}\right)$ where this corresponds to an action-utility profile sequence $\left\{a\left(s^{\tau} \mid s^{t}\right), w\left(s^{\tau} \mid s^{t}\right)\right\}_{\tau=t}^{\infty}$; it is self-enforcing if it satisfies both feasibility and (2) for every date and history $\tau \geq t$ succeeding $s^{t}$. We define the set of self-enforcing agreements as $\mathcal{G}$. Because of our Markov assumption and because all the self-enforcing constraints are forward looking and the time-horizon is infinite the set of self-enforcing agreements depends only on the current state $s$ at a particular date $t$ and is independent of the history $s^{t}$. We shall denote this set of self-enforcing agreements given current state $s$ by $\mathcal{G}_{s}$. Associated with each $\Gamma\left(s^{t}\right) \in \mathcal{G}_{s_{t}}$ are the discounted payoffs to the two agents 
$\left(V_{1}\left(s^{t}\right), V_{2}\left(s^{t}\right)\right)$ given in equation (2). We shall let $\mathcal{V}$ denote the set of payoffs $\left(V_{1}, V_{2}\right)$ which correspond to self-enforcing agreements $\Gamma$, and $\mathcal{V}_{s_{t}}$ denote the set of equilibrium payoffs $\left(V_{1}\left(s^{t}\right), V_{2}\left(s^{t}\right)\right)$.

The sets $\mathcal{G}$ and $\mathcal{V}$ are not necessarily convex because of the presence of $a\left(s^{t}\right)$ on the right hand side of equation (2). This potential non-convexity does not affect our main characterization results and therefore we do not impose further restriction on the model to guarantee convexity. ${ }^{25}$ We define the Pareto-frontier of the payoff $\Lambda(\mathcal{V})$ in the obvious way as the set of payoffs in $\mathcal{V}$ which are not Pareto-dominated and use $\Lambda\left(\mathcal{V}_{s}\right)$ to denote the Pareto-frontier in state $s$. As our objective is to characterize the set of Pareto-efficient self-enforcing agreements (when looked at from the outset of the game) we shall be interested in the set $\Lambda(\mathcal{V})$. We shall say that agreements that correspond to this Pareto-frontier are optimum or optimum contracts and refer to the corresponding actions as optimum actions.

\section{RESULTS}

This section provides the main results of the paper. The existence of optimum contracts is established in Section 3.1. Section 3.2 considers the relationship between the optimum and Nash and conditionally efficient actions and Section 3.3 proves the backloading principle. The long-run properties are examined in Section 3.4 and Section 3.5 which show convergence to a stationary phase which maximizes surplus amongst all self-enforcing contracts. Finally Section 3.6 will consider an example with no uncertainty to illustrate our results. The case where only one agent contributes to output is considered in Section 4.

\subsection{Existence}

We first establish that optimum self-enforcing contracts do exist. This follows from a straightforward argument showing that the equilibrium payoff set is compact.

LEMMA 4 The set of pure-strategy subgame perfect equilibrium payoffs $\mathcal{V}$ is non-empty and compact. Hence optimum contracts exist.

We shall say that a self-enforcing contract is trivial if $a\left(s^{t}\right)=0$ for all $s^{t} \cdot{ }^{26}$ From Assumption 1(i) on the production function, it follows that this corresponds to a point $(0,0) \in \mathcal{V}$. Lemma 4 does not imply the existence of an optimum

\footnotetext{
${ }^{25}$ There are special cases where the sets are convex. The additive production technology case is one such example.

${ }^{26}$ In the next section it is shown that in any optimum self-enforcing contract the actions are never below the Nash equilibrium actions of the breakdown game. Thus if the latter are positive a non-trivial contract will exist although it may be trivial in the sense that it is equal to the repetition of the Nash equilibrium of the break-down game.
} 
non-trivial contract so that it is possible that $\Lambda(\mathcal{V})=(0,0)$ and all our results will apply (trivially) in this case.

\subsection{Actions at a particular date}

We shall consider the dynamic path of actions in sections 3.3 and 3.5 but in this section we consider actions at a given date and how they relate to the Nash best-response and conditionally efficient actions. Our method is to argue by contradiction, changing an assumed optimal contract at a particular date after a particular history. If this change satisfies the self-enforcing and feasibility constraints for both agents at that date, and a Pareto-improvement has been generated, then all prior self-enforcing constraints also hold as by construction the future utility entering these constraints has not been decreased. Equally all future constraints must continue to hold. Hence this leads to a Pareto-superior contract - contrary to the assumed optimality of the original contract.

As we are considering only a particular date we shall, in what follows, suppress the history $s^{t}$ or state $s_{t}$ where there is no ambiguity, and we shall refer to the current state as $s$ and use $r$ to index the state next period where necessary. We first show that actions cannot be below the Nash best-response functions, $a_{i}^{N}\left(a_{j}\right)$ of the breakdown game. The intuition is that if any agent's action is below the Nash best-response action, that agent's action can be increased and surplus divided in such a way that neither agent has an incentive to move to the breakdown and that the increase in action will increase output and utility. Specifically, suppose that $a_{2}$ is below the reaction function (but $a_{1}$ is not). As agent 2's action is increased, because of the hold-up nature of the problem, this will increase agent 1's deviation payoff; suppose however we give agent 1 extra consumption equal to the increase in her deviation payoff to stop her reneging. Since this will be approximately that part of extra output she can appropriate, giving the remainder to agent 2 gives him at least what he would get from increasing his action in the breakdown game. Because he is below his optimal breakdown action this will increase his utility too. Hence both agents can be made better off. In the following statement, recall that $a^{N E}$ is the dominant Nash equilibrium of the breakdown game.

LEMMA 5 In any optimum self-enforcing contract, after any positive probability history $s^{t}, a_{i} \geq a_{i}^{N}\left(a_{j}\right)$, and $\left(a_{1}, a_{2}\right) \geq a^{N E}$.

We would also like to say how the optimum actions relate to the conditionally efficient actions. This is less clear cut as we shall show that optimum actions can be above or below the conditionally efficient actions. We can, however, show that an agent only 'underinvests' (action below the conditionally efficient level) if the other agent's self-enforcing constraint binds and only 'overinvests' if she is at her subsistence consumption (of zero). 
LEMMA 6 In an optimum contract after any positive probability history, (i) If agent $i$ is unconstrained (i.e., $V_{i}>D_{i}\left(a_{j}\right)$ ), then $a_{j} \geq a_{j}^{*}\left(a_{i}\right)$; (ii) If agent $i$ has positive consumption $\left(c_{i}>0\right)$, then $a_{i} \leq a_{i}^{*}\left(a_{j}\right)$.

REMARK Lemma 6 relates the optimum actions to the conditionally efficient level. It is, unlike Lemma 5, independent of the default structure we have specified (requiring only that deviation payoffs are continuous and non-decreasing in the action of the other agent).

The intuition for the proof is straightforward. If, say agent 1's, self-enforcing constraint is not binding then there is no cost (but a surplus gain) to increasing agent 2's action up to the conditionally efficient level. Equally if an action, say agent 1's action, is above the conditionally efficient level it will be profitable to reduce it (surplus will increase). This cannot tighten agent 2's self-enforcing constraint, and agent 1 is better off since her action has been reduced, so provided agent 2's consumption can be held constant this will lead to a Pareto-improvement. But aggregate output has fallen so aggregate consumption must be cut. If agent 1 has positive consumption it will be possible to reduce her consumption to compensate agent 2 . However, if agent 1 already has zero consumption then she cannot compensate agent 1 and a Pareto-improvement may not be possible.

There are two straightforward implications of Lemma 6(ii). Firstly it is impossible in an optimum contract that both agents overinvest (they cannot both have zero consumption given output is positive). Secondly an agent cannot be permanently overinvesting (i.e., with probability one) as this would imply that her consumption is always zero, which cannot be self-enforcing.

\subsection{Backloading}

As discussed in the introduction there is a well known backloading principle that applies when commitment by one agent is limited. This principle says that ceteris paribus transfers to the agent who cannot commit should be backloaded into the future if the commitment constraint is binding, to provide a carrot in the future that would be forgone if the agent reneged. The operation of this principle in our environment where both agents undertake an action or investment and neither can commit is more subtle, as discussed earlier. Nevertheless we shall show that backloading does apply in a way we make precise below and moreover has the additional implication that one agent may overinvest in the early periods of an optimum contract.

We begin by showing (in the next lemma) that, under certain conditions, if an agent is allocated all the current output then this is sufficient to guarantee that the self-enforcing constraint of that agent is not violated. The basic idea is straightforward: if an agent is getting all of current output then there is no shortrun gain to defaulting as no matter how big a portion of output can be claimed in breakdown. However, care has to be taken with this argument because the 
agent may be able to choose a more advantageous action in the breakdown. Thus, in more detail, suppose agent 2 gets allocated all the current output. Consider starting from agent 2's best-response (in the breakdown game) to agent 1's action, and hold the latter fixed; by definition agent 2 cannot benefit by deviating at this point; if agent 2's action is increased and he is still being allocated all output, then his utility will be rising until his conditionally efficient action is reached, where his payoff is maximized. So provided $a_{2}$ is above or equal to his best-response, but not higher than the conditionally efficient action, he is weakly better off at $a_{2}$ getting all consumption than best responding-no matter how much he can claim of the output in the breakdown. Hence the self-enforcing constraint is satisfied, even if deviating leads to no future losses (i.e., even if agent 2's future payoff is as low as it can be). This property is important for our backloading result as it will enable us to verify that the self-enforcing constraint for an agent is satisfied by checking that that agent is receiving all the available output. ${ }^{27}$ Recall that the deviation continuation payoff $D_{2}\left(a_{1, r}^{N E}, r\right)$ is the payoff agent 2 gets from Nash-reversion starting in state $r$.

LEMMA 7 If in state $s$ (but suppressing $s$ in the notation) $c_{2}=y\left(a_{1}, a_{2}\right)$, $a_{2}^{N}\left(a_{1}\right) \leq a_{2} \leq a_{2}^{*}\left(a_{1}\right)$ and $V_{2, r} \geq D_{2}\left(a_{1, r}^{N E}, r\right)$, all $r \in \mathcal{S}$, then

$$
c_{2}-a_{2}+\delta \sum_{r \in \mathcal{S}} \pi_{s r} V_{2, r} \geq D_{2}\left(a_{1}\right)
$$

moreover the inequality is strict if $a_{2}>0$. Likewise with the agent indices swapped.

We now present our main backloading result. For notational convenience we will treat actions and consumptions at a particular date as random variables and write $a_{i}^{t}$ and $c_{i}^{t}$ for $a_{i}\left(s^{t}\right)$ and $c_{i}\left(s^{t}\right)$ etc.

THEOREM 1 (i) If at $\tilde{t}$ in an optimum contract (after positive probability history $\left.s^{\tilde{t}}\right)$, agent 1 , say, is unconstrained and $a_{1}^{\tilde{t}}<a_{1}^{*}\left(a_{2}^{\tilde{t}}\right)$, then at all previous dates $t<\tilde{t}$ along the same history, $c_{2}^{t}=0$; (ii) If at $\tilde{t}$ in an optimum contract (after positive probability history $\left.s^{\tilde{t}}\right)$, agent 2 , say, has $a_{2}^{\tilde{t}}>a_{2}^{*}\left(a_{1}^{\tilde{t}}\right)$, then at all previous dates $t<\tilde{t}$ along the same history, $c_{2}^{t}=0$.

The claim of Theorem 1(i) is that if in any optimum contract agent 1 is underinvesting but unconstrained then agent 2 will have been held to his subsistence consumption level (zero) in all previous periods along the history to that point. (Recall that if agent 1 is underinvesting, this is because agent 2 is constrained and any further investment by agent 1 will lead to agent 2 preferring to take the increased deviation payoff.) The idea is that if agent 2 has positive consumption earlier, agent 1's current action can be increased (increasing current surplus)

\footnotetext{
${ }^{27}$ Note this result does not refer to optimum contracts but is a statement about the properties of the deviation payoff function $D_{i}(\cdot)$.
} 
and at the same time consumption can be transferred at the current date to agent 2 to stop him reneging; agent 1 may be worse off currently but can be compensated for her increased effort by agent 2 transferring consumption at the earlier date. Essentially, the backloading of agent 2's consumption allows his later constraint to be relaxed. Since agents are risk neutral they do not care about the timing of consumption flows (keeping the action plans fixed) if the expected discounted value is the same, but the backloading has permitted current surplus to be increased, leading to a Pareto-improvement. It is important though that agent 1 is unconstrained for this result to hold and we will show later that it may not apply if agent 1 is also constrained at the later date.

Theorem 1(ii) demonstrates that if ever agent 2 is overinvesting then consumption has already been backloaded to the maximum extent possible in all previous periods. This makes it clear that the backloading principle extends to actions as well as consumption and that actions may be above the conditionally efficient levels. This however involves an efficiency loss not incurred by backloading consumption. So reducing consumption is more efficient than increasing the action. Nevertheless it may be optimal on the margin to increase the action as for a small increase starting from the efficient level the loss will be of second-order and it will enable the action of the other agent to be increased without violating the self-enforcing constraint and thus actions of one agent may be above the conditionally efficient levels in the early periods of the optimum contract

\subsection{Second-Best Surplus Maximization}

In the next section we shall show that an optimum contract converges to one that maximizes joint utility. In this section we shall first show that the maximization of joint utility involves choosing the actions that maximize current surplus subject to the self-enforcing and feasibility constraints. We begin by defining such actions and also a joint utility maximizing contract.

Definition 1 An action pair $a$ in state $s_{t}$ at date $t$ is current surplus maximizing if $a \in \arg \max _{a \in \Re_{+}^{2}}\left\{y\left(a_{1}, a_{2}, s_{t}\right)-a_{1}-a_{2}: \exists\right.$ a self-enforcing contract $\Gamma\left(s^{t}\right) \in \mathcal{G}_{s_{t}}$ starting at date $t$ with $\left.a\left(s^{t}\right)=a\right\}$.

Definition 2 A self-enforcing contract $\Gamma\left(s^{t}\right) \in \mathcal{G}_{s_{t}}$ at date $t$ in state $s_{t}$ is joint utility maximizing if the sum of the corresponding continuation utilities from $t$ onwards is maximized across all possible self-enforcing contracts: $\left(V_{1}, V_{2}\right) \in$ $\arg \max _{\left(V_{1}, V_{2}\right) \in \mathcal{V}_{s_{t}}}\left(V_{1}+V_{2}\right)$.

Note that a current surplus maximizing $a$ is found by looking across all selfenforcing contracts starting from $s$ and picking one that maximizes the surplus in the first period of the contract, irrespective of what happens later. If a selfenforcing contract can be constructed with current surplus maximizing actions 
at every date, then it is clearly joint utility maximizing. The next result asserts that joint utility maximizing contracts always have this property.

LeMma 8 Any joint utility maximizing self-enforcing contract starting at date $t$ from $s^{t}, \Gamma\left(s^{t}\right)$, has with probability one current surplus maximizing actions at each date $\tau \geq t$.

Changing actions away from the current surplus maximizing ones will lower utility at that particular date and hence overall utility. What the lemma shows is that there is no additional benefit in terms of relaxing one of the self-enforcing constraints.

We show next that if at any point the self-enforcing constraints bind for both agents and there is no over-efficiency of actions, then the optimum contract always involves joint utility maximization from the next period onward. Hence from Lemma 8 this involves the surplus maximizing actions at every subsequent date.

LEMMA 9 If in an optimum contract $s^{t}$ has positive probability, the self-enforcing constraints of both agents bind at $t$, and $a_{i}^{t} \leq a_{i}^{*}\left(a_{j}^{t}\right), i, j=1,2, i \neq j$, then the contract must specify joint utility maximization from $t+1$ (i.e., in every positive probability successor state).

The idea behind Lemma 9 is that if it were the case that in some successor state $r$ joint utility was not maximized, then it would be possible to replace $\left(V_{1, r}, V_{2, r}\right)$ by $\left(\hat{V}_{1, r}, \hat{V}_{2, r}\right) \in \mathcal{V}_{r}$ such that $\hat{V}_{1, r}+\hat{V}_{2, r}>V_{1, r}+V_{2, r}$ and demonstrate a Paretoimprovement. To show this it is necessary that both agents were previously constrained. If either agent were unconstrained then replacing $V_{i, r}$ with $\hat{V}_{i, r}$ might lower utility for one agent.

\subsection{Convergence}

We now present the result that an optimum contract has actions which converge almost surely to current surplus maximizing actions (and, a fortiori, the contract converges to a joint utility maximizing one). To show this, we first show that there exists a stopping time which is finite almost surely such that both $c_{1}>0$ and $c_{2}>0$ at some point before this time. The argument is intuitive: provided at least one agent has a strictly positive payoff, then one agent, say agent 1 , must take a positive action at some point. Thus agent 1 must receive positive consumption at some point not too far after the action was taken, or else her overall payoff would be negative, something which is inconsistent with selfenforcement (an agent can always guarantee herself at least zero by taking a null action each period). Likewise, by the fact that agent 1 took a positive action, agent 2 can get a positive share of that output by the hold-up assumption on the breakdown payoffs, and hence must have positive continuation utility at this 
point. Thus agent 2 must also anticipate positive consumption. This situation happens repeatedly in an optimum contract, and thus positive consumption for both agents occurs with probability one (the proof is only complicated by the need to ensure that the number of periods before positive consumption is received is bounded independently of the current continuation utility is divided). Next, once both agents have had positive consumption, our backloading results imply that there cannot be overinvestment, and if either agent is unconstrained then actions are at the efficient level. Alternatively, if both agents are constrained, we know that joint utility maximization occurs thereafter by the previous lemma.

THEOREM 2 For any optimum contract, with probability one there exists a random time $T<\infty$ such that for $t \geq T$, $a^{t}$ is current surplus maximizing.

Suppose that both agents have had positive consumption at some date prior to date $\hat{t}$. Then from Theorem 1(ii), for $t \geq \hat{t}, a_{i}^{t} \leq a_{i}^{*}\left(a_{j}^{t}\right)$ for $i=1,2$. However, if agent $i$ is unconstrained at date $t$, then it follows from Theorem 1(i) that $a_{i}^{t} \geq a_{i}^{*}\left(a_{j}^{t}\right)$, otherwise the consumption of agent $j$ could not have been positive prior to date $\hat{t}$. Equally from Lemma $6(\mathrm{i}), a_{j}^{t} \geq a_{j}^{*}\left(a_{i}^{t}\right)$ as there is no need to hold agent $j$ 's action below the efficient level if agent $i$ is unconstrained. So if one agent is unconstrained, both actions are efficient. We can conclude that inefficiency can only occur after date $\hat{t}$ when both agents are constrained. We thus have the following corollary.

COROLlary 1 For any optimum contract, with probability one there exists a random time $T<\infty$ such that for $t \geq T$, and for any state $s_{t} \in \mathcal{S}$ in which efficiency $a^{* *}$ is not achievable for any division of the surplus (i.e., for which the current surplus maximizing actions are inefficient), then both self-enforcing constraints bind and there is no overinvestment by either agent.

The next theorem considers the canonical two-sided action case in which both actions are always positive. This allows us to present the sharpest results in terms of optimum action levels relative to unconstrained efficient levels. It shows there will be two phases, one (which may not exist depending on how ex ante surplus from the relationship is divided) is a backloading phase with zero consumption and overinvestment by one of the agents (the same agent throughout the phase), and the other phase (which exists with probability one) will have no overinvestment, but consists of a possible initial transition period which is then followed by either efficient actions, if they can be sustained in equilibrium in that state, or otherwise by both constraints binding and positive consumption.

TheOREM 3 Whenever the Nash actions $a_{i, s}^{N E}$ are positive, $i=1,2$, all $s \in \mathcal{S}$, then there exists $i \in\{1,2\}$ and a random time $\tilde{t}, \infty>\tilde{t} \geq 0$ with probability one, such that an optimum satisfies a.s.:

Phase 1: $c_{i}^{t}=0, a_{i}^{t}>a_{i}^{*}\left(a_{j}^{t}\right)$ and $a_{j}^{t} \leq a_{j}^{*}\left(a_{i}^{t}\right)$, for $0 \leq t<\tilde{t}, j \neq i$; 
Phase 2: $a_{1}^{t} \leq a_{1}^{*}\left(a_{2}^{t}\right)$ and $a_{2}^{t} \leq a_{2}^{*}\left(a_{1}^{t}\right)$ for $t \geq \tilde{t}$, and after the first period of phase 2, if $s_{t}$ is such that $a^{* *}$ is current surplus maximizing (i.e., can be sustained in equilibrium) then $a^{t}=a^{* *}$; otherwise for both agents, $i=1,2$, the incentive constraint binds, $a_{i}^{t}<a_{i}^{*}\left(a_{j}^{t}\right) \leq a_{i}^{* *}, j \neq i$, and $c_{i}^{t}>0$.

The requirement of positive Nash actions is a simple way to ensure that optimum actions at each date are positive by virtue of Lemma 5 . We need to assume this to prove Theorem 3 for two reasons. Firstly, even if both actions are productive, it may be that overinvestment does not occur in the backloading phase. This might be the case if $a_{i}=0$ and $a_{i}^{*}\left(a_{j}\right)=0$ and the marginal product at zero is well below one. In this case the optimum action may be at the corner solution where the marginal product is below one and the optimum action is zero. Secondly, underinvestment may not occur in the second phase as it possible that the efficient action levels are zero.

If current surplus is not maximized after the first period of Phase 2, then $a \neq a^{* *}$ but by Theorem 3 this implies both constraints bind, in which case current surplus is maximized thereafter. Hence we have the following corollary.

COROLlARY 2 With probability one, current surplus is not maximized in at most two periods of Phase 2. ${ }^{28}$

\subsection{Example with No Uncertainty}

We consider a simple example with no uncertainty to illustrate our results and demonstrate that the backloading phase of Theorem 3 with overinvestment can exist for some initial distribution of continuation utilities. The example has an additive production technology and is similar to the model of joint production in Garvey (1995). ${ }^{29}$ We assume the production function is

$$
y\left(a_{1}, a_{2}\right)=f_{1}\left(a_{1}\right)+f_{2}\left(a_{2}\right)=2 b \sqrt{a_{1}}+2 \sqrt{a_{2}}
$$

for a parameter $b \in(0,1)$. For simplicity the breakdown payoffs are of the form $\phi_{i}\left(a_{1}, a_{2}\right)=\theta_{i 1} f_{1}\left(a_{1}\right)+\theta_{i 2} f_{2}\left(a_{2}\right)$ where the parameters are $\theta_{11}=\theta_{22}=0$ and $\theta_{12}=\theta_{21}=1$. Thus in the breakdown both agents can grab all of the other agent's output but if they do so they lose their own output. The conditionally efficient actions are independent of the other agent's action and given by $a_{1}^{*}=b^{2}$ and $a_{2}^{*}=1$ and maximal efficient surplus is $1+b^{2}$. Given the additive technology

\footnotetext{
${ }^{28}$ In the case of additive production technology this can be strengthened to current surplus being maximized in all but the first period of Phase 2 .

${ }^{29}$ Garvey (1995) has linearly additive outputs and quadratic cost functions but this is equivalent to our formulation with square-root production functions and linear investment costs. His concern is with finding a legal structure, joint ventures or integration, that is best suited (in terms of a minimum discount factor) to sustaining the efficient investment levels. He does not therefore examine the main concern addressed here, namely the temporal structure of investments.
} 
the Nash best-response functions are dominant strategies, $a_{1}^{N}=a_{2}^{N}=0$ so the best response is no investment. ${ }^{30}$ Hence the deviation payoffs are $^{31}$

$$
D_{1}\left(a_{2}\right)=(1-\delta) 2 \sqrt{a_{2}} \quad \text { and } \quad D_{2}\left(a_{1}\right)=(1-\delta) 2 b \sqrt{a_{1}} \text {. }
$$

For illustration we consider the special case of $\delta=1 / 3$ and $b=\sqrt{3} / 3$. $^{32}$ The solution can be found by first deriving the efficiency stationary solution and then working backwards from the stationary solution to construct the entire Pareto-frontier in a similar fashion to that described in Thomas and Worrall (1994). ${ }^{33}$ It should be stressed that the procedure used here computes the exact solution and does not rely on numerical methods.

The solution is illustrated in Figure 1 which plots some of the variables of interest against the (continuation) utility $V_{1}$, of agent 1 . The stationary solution is indicated by point " $\mathrm{S}$ ". Figure 1(a) illustrates the Pareto-frontier. The left hand endpoint of the frontier is determined at the point where this function has a zero slope and the right hand endpoint where the slope is infinite and $a_{1}=0$. The stationary solution is where joint utility, $V_{1}+V_{2}$, is maximized so that the slope of the frontier is -1 at point S. Figure 1(b) illustrates the net surplus for different values of $V_{1}$ indicating that net surplus is maximized at the stationary solution. Figure 1(d) illustrates that agent 2 is always underinvesting $\left(a_{2}<a_{2}^{*}=1\right)$ for all $V_{1}$. Agent 1 is always constrained but as $V_{1}$ is increased $a_{2}$ can also be increased without agent 1 reneging. Figure $1(\mathrm{c})$ shows that agent 1 's investment is efficient $\left(a_{1}^{*}=1 / 3\right)$ for $V_{1} \in[2 / 27,4 \sqrt{21} / 27]$ and that agent 1 overinvests (and has zero consumption) for low values of $V_{1}<2 / 27$ and underinvests for larger values of $V_{1}$ and at the stationary solution. If we suppose that the initial distribution of utilities is such that $V_{1}<2 / 27$ then the optimum contract evolves in the following way. In the initial period agent 1 has zero consumption and overinvests. In the next period it can be shown that the continuation value for $V_{1}$ will be in the range $(2 / 27,4 \sqrt{21} / 27)$. Thus next period the action $a_{1}$ is chosen efficiently, $a_{1}=1 / 3$. In the periods thereafter the continuation values are the utility maximizing ones at the stationary point where both agents underinvest. Thus in this example and for the parameters we've used, and for an an initial distribution where $V_{1}<2 / 27$,

\footnotetext{
${ }^{30}$ Strictly Theorem 3 does not apply since the Nash equilibrium actions are zero. However, the importance of the assumption of positive Nash equilibrium actions was to rule out trivial contracts but such contracts are not optimal for the parameter values chosen and hence the substance of the theorems does apply.

${ }^{31}$ For the purposes of calculating the example all per-period payoffs have been multiplied by $(1-\delta)$.

${ }^{32}$ In this example a non-trivial contract is sustainable for any $\delta>0$ and an efficient stationary solution is sustainable if $\delta \geq 1 / 2$. The value $\delta=1 / 3$ is chosen below this critical value so that the efficient outcome is not sustainable in the the stationary solution but large enough to generate simple but interesting dynamics for the optimum contract. The value of $b$ is simply chosen for convenience.

${ }^{33}$ Details of the calculation are available on request.
} 


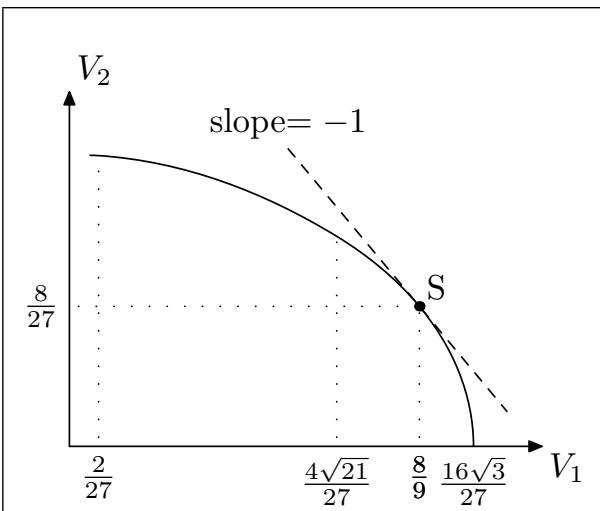

(a) Pareto-frontier

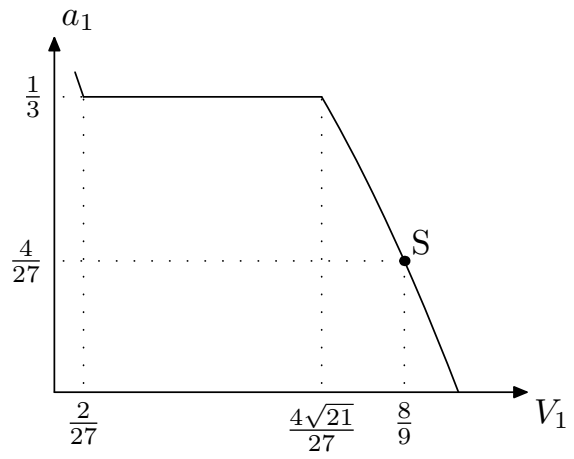

(c) ACtion of Agent 1.

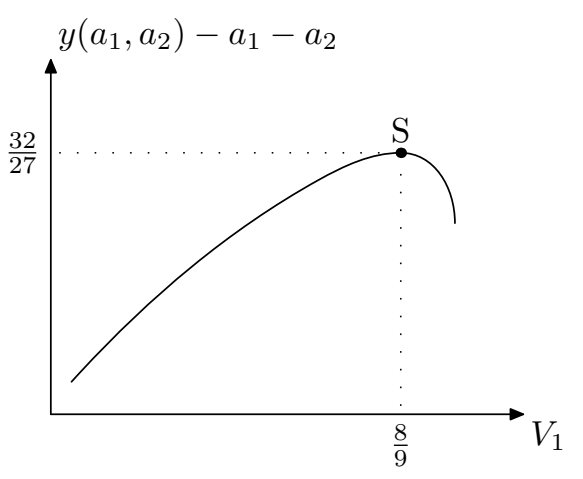

(b) Net surplus.

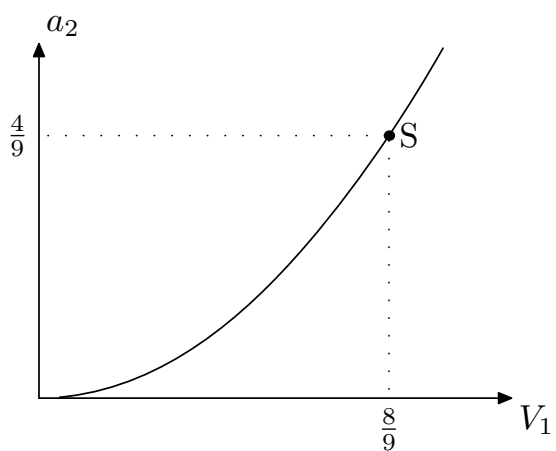

(d) Action of Agent 2.

Figure 1: EXAMPLE WITH $\delta=\frac{1}{3}$ AND $b=\frac{\sqrt{3}}{3}$.

there is at most one period of Phase 1 where there is overinvestment and after two periods the stationary solution is reached where both agents are underinvesting. ${ }^{34}$

\section{ONE-SIDED INVESTMENT}

In this section we discuss the case where only one of the two agents makes an investment which has been the subject of most of the previous literature (see, e.g., Albuquerque and Hopenhayn, 2004; Thomas and Worrall, 1994). We shall show that in this case there is no overinvestment by the contributing agent. ${ }^{35} \mathrm{We}$

\footnotetext{
${ }^{34}$ For different parameter values there may be more than one period of Phase 1 in which there is overinvestment.

${ }^{35} \mathrm{~A}$ difference arises in the one-sided and two-sided cases since when only one agent takes any action it is not always possible to adjust actions to smoothly raise or lower the continuation utilities for both agents as is the case with the two-sided case.
} 
suppose that this is agent 1 and assume that agent 2 never contributes towards output. We therefore write output as $y\left(a_{1}, a_{2}, s_{t}\right)=f\left(a_{1}, s_{t}\right)$ and the breakdown payoff for agent 1 as $\phi_{1}\left(a_{1}, s_{t}\right) \leq f\left(a_{1}, s_{t}\right)$. In this case agent 1 's self-enforcing constraint reduces to a more conventional nonnegative surplus constraint,

$$
V_{1}\left(s^{t}\right) \geq D_{1}\left(s_{t}\right)=\phi_{1}\left(a_{1}^{N E}\left(s_{t}\right), s_{t}\right)-a_{1}^{N E}\left(s_{t}\right)+\delta \sum_{s_{t+1} \in \mathcal{S}} \pi_{s_{t} s_{t+1}} D_{1}\left(s_{t+1}\right)
$$

and the following can be proved.

THEOREM 4 In the case of one-sided investment where, say, agent 1 is the only contributor to output, then at any date $t$ and state $s, a_{1}^{t} \leq a_{1}^{*}$; i.e., overinvestment never occurs in an efficient self-enforcing contract.

This result is perhaps unsurprising in view of the idea that backloading of utility will only apply to agent 2 , the agent whose self-enforcing constraint can prevent efficient actions by agent 1 . Overinvestment (and hence $c_{1}=0$ ) implies a negative current utility for agent 1 , and as the future goes against agent 1 , this would lead to a negative overall utility, something which would violate agent 1's constraint.

\section{CREDIT CONSTRAINTS}

In this section we shall assume that agents can save in a private account at a fixed net rate of interest $r{ }^{36}$ We shall however assume that agents are credit constrained and cannot borrow. The issue addressed is whether by using savings it may be possible to achieve utility distributions at future dates that cannot be achieved without savings. For example, if agent 1 saves some of her early transfers and then at date $t$ makes a transfer to agent 2 from her savings, agent 2's continuation utility at $t$ will be higher by the amount of the savings transfer (holding all future transfers and actions constant), and agent 1's utility correspondingly lower. Potentially, provided this transfer is credible, it may allow more efficient actions to be undertaken at date $t$, allowing an ex ante Paretoimprovement. We show that provided $\delta(1+r) \leq 1$, that is if the interest rate is less than the discount rate, this is not possible. ${ }^{37}$ Intuitively, we have seen that at most one agent has utility backloaded for incentive reasons; this suggests that there is no gain if the other agent saves. ${ }^{38}$

\footnotetext{
${ }^{36}$ An alternative - leading to different results - would be to assume an escrow account held by a trusted third agent where agents lose access to the account if they default. We assume here that such accounts are ruled out.

${ }^{37}$ If $\rho:=(1-\delta) / \delta$ is the discount rate, then $\delta(1+r) \leq 1$ is equivalent to $r \leq \rho$. With this assumption savings cannot change feasible payoffs and if there is any saving it will be for incentive reasons. The previous model without savings corresponds to the situation where $r=-1$.

${ }^{38} \mathrm{~A}$ similar result is obtained by Itskhoki (2007) in a general model with one-sided commitment.
} 
We change the model as follows. Let $S_{i}^{t}$ denote agent $i$ 's total saving at the start of period $t$, including interest up to period $t$ (in general this depends on the history $s^{t-1}$ but this dependence is left implicit in the notation). Borrowing is ruled out so $S_{i}^{t} \geq 0$, with $S_{i}^{0}=0 .{ }^{39}$ At the division stage, let $\chi_{i}\left(s^{t}\right)$ denote the amount of resource transferred to agent $i$ after history $s^{t}$ (this need no longer coincide with consumption $\left.c_{i}\left(s^{t}\right)\right)$, subject to the constraints that $\chi_{1}\left(s^{t}\right)+\chi_{2}\left(s^{t}\right) \leq y\left(a\left(s^{t}\right), s_{t}\right)$ and $-S_{i}^{t} \leq \chi_{i}\left(s^{t}\right) \leq S_{j}^{t}+y\left(a\left(s^{t}\right), s_{t}\right)$, for $i=1,2$, and $i \neq j$. Thus it is possible to receive more than current output if the other agent-whose transfer would be negative - contributes from their savings. We are assuming then that transfers from savings occur at the same time that output is divided (although making this subsequent to output division would not affect the results). ${ }^{40}$ Thereafter, at a new decision point, each agent simultaneously chooses consumption $c_{i}\left(s^{t}\right)$, subject to $0 \leq c_{i}\left(s^{t}\right) \leq S_{i}^{t}+\chi_{i}\left(s^{t}\right)$, so that savings at the start of $t+1$ are $S_{i}^{t+1}=(1+r)\left(S_{i}^{t}+\chi_{i}\left(s^{t}\right)-c_{i}\left(s^{t}\right)\right)$. We assume that the consumption decisions are observable (although it turns out that nothing depends on this). A contract now specifies $\left(a_{i}\left(s^{t}\right), \chi_{i}\left(s^{t}\right), c_{i}\left(s^{t}\right)\right)$ for each history $s^{t}$ and $i=1,2$. We extend the definitions of equilibrium etc. in the obvious way. It is useful to let

$$
U_{i}\left(s^{t}\right)=\chi_{i}\left(s^{t}\right)-a_{i}\left(s^{t}\right)+\mathrm{E}\left[\sum_{\tau=t+1}^{T} \delta^{\tau-t}\left(\chi_{i}\left(s^{\tau}\right)-a_{i}\left(s^{\tau}\right)\right) \mid s^{t}\right]
$$

denote the discounted value of the contracted transfers net of action costs. As before

$$
V_{i}\left(s^{t}\right)=c_{i}\left(s^{t}\right)-a_{i}\left(s^{t}\right)+\mathrm{E}\left[\sum_{\tau=t+1}^{T} \delta^{\tau-t}\left(c_{i}\left(s^{\tau}\right)-a_{i}\left(s^{\tau}\right)\right) \mid s^{t}\right]
$$

denotes the continuation utility from consumption.

If agent $i$ chooses to default at $t$, then she will retain her savings (and can do no better than consume them immediately) so that $S_{i}^{t}$ is added to the deviation payoff. Thus the incentive constraint at $t$ will be

$$
V_{i}\left(s^{t}\right) \geq D_{i}\left(a_{j}\left(s^{t}\right), s_{t}\right)+S_{i}^{t} .
$$

However, because $\delta(1+r) \leq 1$, discounted current and future consumption cannot exceed expected discounted net transfers from $t$ on plus current beginning

\footnotetext{
${ }^{39}$ We can allow initial resources to be positive, $S_{i}^{0}>0$, and the equivalence result established below still goes through (the equilibrium without savings will however differ slightly from that studied earlier).

${ }^{40}$ To be precise, we could spell out the post production timing as follows. First, the Nash demand game takes place, followed by simultaneous transfers by each agent to the other out of savings (and then finally consumption decisions are taken). We only need to focus on the net transfer $\chi_{i}\left(s^{t}\right)$, however, as if (and only if) the incentive constraint specified below holds, it will be an equilibrium to implement the transfer. Specifically, $i$ 's Nash demand is $\chi_{i}\left(s^{t}\right)$ whenever $0 \leq \chi_{i}\left(s^{t}\right) \leq y\left(a\left(s^{t}\right), s_{t}\right)$, and otherwise 0 or $y\left(a\left(s^{t}\right), s_{t}\right)$, as appropriate, while the direct transfer from $i$ to $j$ equals $\max \left\{-\chi_{i}\left(s^{t}\right), 0\right\}$. Again, the fine details of the process are unimportant so long as a failure to adhere to the equilibrium contract triggers breakdown.
} 
of period savings, that is, ${ }^{41} V_{i}\left(s^{t}\right) \leq U_{i}\left(s^{t}\right)+S_{i}^{t}$. Notice that savings per se effectively cancel out of the incentive constraint, but the issue is whether by allowing flexibility with respect to timing of transfers better allocations can be attained.

To argue that savings will not matter we start from an optimum contract in the model with savings and replace it with a contract which has no transfers out of savings. As an example, suppose a single transfer is made, out of savings, from agent 2 to agent 1 at time $t$ after some history $s^{t}$ (agent 1 also gets all of current output at $t$ ). In this case make the following changes: cut the transfer (i.e., the dissaving) from agent 2 to zero, but leave agent 1 with all of current output. So at $t$, agent 1's continuation utility is lowered by the lost transfer. Next attempt to compensate agent 1 by increasing her transfer out of output, at agent 2's expense, at the immediately preceding date and history. If agent 1 is fully compensated then there are no further changes. If she is not fully compensated even when all output is allocated to her at $t-1$, then we carry forward the remaining lost utility to $t-2$, and so on. Full compensation (in terms of discounted transfers) must be possible by date 0 . This is because in the original allocation, agent 2 was able to accumulate the savings from the output allocated to him before the period $t$ transfer to agent 1 , so by shifting all output to agent 1 at previous dates, agent 1 must be at least as well off-she would be directly receiving all the transfers which agent 2 had used to accumulate his savings. These changes satisfy three important properties: (1) they do not affect the initial distribution of utility (more precisely, of discounted transfers, the $U_{i}\left(s^{0}\right)$ ), (2) transfers are made nonnegative (so that savings do not need to be used), and (3) at any history, either agent $i$ is no worse off than in the initial allocation or agent $i$ receives all of current output. The point of (3) is that even if an agent is made worse off, because she receives all of current output she will not want to renege (appealing to Lemma 7). Thus the ability to transfer savings is not needed to support the current actions even though an agent may be worse off at some points in the future. That is, the same actions and initial utilities can be supported when savings are not possible.

Generalizing this approach to allow for transfers out of savings at multiple dates and after any history, the following result can be proved.

TheOREM 5 The Pareto-frontier in the model with savings coincides with that in the model without savings.

\footnotetext{
${ }^{41}$ If all transfers are non-negative then $V_{i}\left(s^{t}\right)$ is maximized if they are consumed immediately, and we have $V_{i}\left(s^{t}\right)=U_{i}\left(s^{t}\right)+S_{i}^{t}$. If there are negative transfers, some earlier transfers must be shifted via saving until later; moving resources backwards in time cannot increase their discounted value.
} 


\section{DISCUSSION OF ASSUMPTIONS}

In this section we discuss to what extent our results generalize to different assumptions about the way the surplus division, breakdown and subsequent punishments were modeled, and the relationship to the existing literature.

First, consider the timing of actions, surplus division and breakdown. We assumed that actions are taken, and observed, before the agents decide on their demands in the Nash demand game; however this is equivalent in terms of the subgame perfect equilibria to a model in which actions and demands are determined simultaneously. More importantly, in our formulation action deviations can only be punished by triggering the breakdown payoffs at the surplus division stage. This is consistent with the approach in the existing literature, including where multiple agents take actions (see, e.g., Halonen, 2002; Rayo, 2007). An alternative formulation would be to assume that a deviation at the action stage can be punished independently of going to the breakdown payoffs after output is realized. The idea would be that an action deviation is observed immediately by the other agent who may be able to take measures that affect output or breakdown payoffs (i.e., "putting a spanner in the works"). If this was severe enough, for example, if output could be credibly reduced to zero, the relevant incentive constraints may concern only the division stage. Such a formulation, by making a deviation at the action stage more easily punishable would make it easier to support efficient actions than in the current setup. ${ }^{42}$

Secondly, the idea of going immediately to breakdown if there is a deviation (rather than, say, starting renegotiation) follows most of the literature, and is in the spirit of repeated game analysis in which deviations from agreed courses of action are punished with severe continuations. In general, of course, renegotiationproofness may not be satisfied in our model. ${ }^{43}$ Note that because agents can always opt for breakdown, our incentive constraint that equilibrium utilities can be no lower than breakdown payoffs must be satisfied by any equilibrium approach, so we are allowing for the widest possible set of equilibria (subject to the third point, below).

Thirdly, we assumed that in periods subsequent to a deviation, the Nash equilibrium of the breakdown game is played. If there are multiple Nash equilibria, we assumed it is the Pareto-dominant one that is selected, but this could be any of them, and it is only necessary to assume that the continuation equilibrium selected is fixed in each state. Likewise reversion to the worst subgame perfect continuation, which may be more severe than what we are assuming, would not

\footnotetext{
${ }^{42} \mathrm{~A}$ number of papers in the relational contract literature examine the case where actions are unobservable as well as unverifiable (see, e.g., Baker et al., 2002; Levin, 2003; Rayo, 2007), so that immediate punishment of action deviations is anyway not feasible.

${ }^{43}$ The few papers which have considered renegotiation-proofness in the one-sided context (e.g., Kovrijnykh, 2009; Opp, 2010; Thomas and Worrall, 1994) have generally shown that although restricting the set of efficient contracts, renegotiation-proofness does not change the basic backloading property of the efficient contract. When surplus is destroyed in going to the breakdown, renegotiation would have more bite than in the existing literature.
} 
affect the results, ${ }^{44}$ although in the existing models in this area the two coincide. Of course, more severe punishments will allow a larger range of equilibria to be sustained.

Alternatively the deviation payoffs could be derived from different assumptions about the nature of the breakdown game. For example, if there are state-dependent nonnegative outside options which can only be taken after the end of the current period $t$ (so agents are locked in for the current period after observing the state), and they are no better in expectation from $s_{t}$ than the (best) Nash-reversion payoffs $D_{i}\left(a_{j}^{N E}\left(s_{t+1}\right), s_{t+1}\right)$, then the characterization we give will still apply. See Bond (2003) for a model of this type in a related context.

Fourthly, we used a Nash demand game to model the surplus division stage. In most of the existing literature the issue does not arise as it is usually assumed that existing property rights specify claims over, or allocate shares of, total output (in many cases it is assumed that one agent has control over all the output). There is then a subsequent stage, after output is realized, where agents decide on transfers to the other agent or agents out of their share. Deviation at the transfer stage leads to the agents being left with their existing output shares. Expressed in our framework, this occurs when $\sum_{i} \phi_{i}\left(a, s_{t}\right)=y\left(a, s_{t}\right)$, for all $a$ so that the breakdown payoffs exhaust the surplus. In this case we do not need the Nash demand game; an equivalent way (in terms of equilibrium payoffs that arise) of modeling the division stage would be - as in the existing literature - to suppose that each agent $i$ actually receives her $\phi_{i}\left(a, s_{t}\right)$ when output is realized, and then there is a subsequent stage where agents simultaneously make voluntary transfers to each other. In general, however, ownership over output may not be so well defined, and we want to allow for surplus destruction to occur when property rights are enforced, so that the $\phi_{i}\left(a, s_{t}\right)$ sum to less than output. In this case, some mechanism is needed to determine how output is split. In the spirit of relational contracting, our approach is simply to capture the idea that a deviation from the implicit contract will trigger breakdown. As we stressed, the Nash demand game is one simple way of formalizing this, but any extensive form for which it is credible to use breakdown as a punishment for any deviation will have the same set of equilibrium payoffs. This will generally be the case whenever both agents retain the option to go to breakdown until an agreement on division is struck. ${ }^{45,46}$

\footnotetext{
${ }^{44}$ Mutatis mutandis, so that all definitions involving future punishment payoffs incorporate the change.

${ }^{45}$ The story can be made more compelling by amending the model slightly so that if an agent unilaterally triggers the default then she does epsilon better than if both do, or if only the other agent triggers default. Thus each has a strong incentive to "run to the lawyer" if there is a suspicion that the other agent may do so, as there would be after a deviation.

${ }^{46} \mathrm{An}$ alternative is to interpret the breakdown payoffs as a reduced form for the outcome of bargaining when disagreement occurs. For example, if one agent is able to make a binding takeit-or-leave-it offer to the other about the split of current surplus before recourse to breakdown is possible, then the outcome of this would yield the appropriate deviation payoffs. Provided
} 
Finally, a difference between our approach and some of the literature is that we treat the breakdown payoffs as fixed. A number of papers in the relational contracting and property rights tradition assume that the breakdown payoffs are a consequence of the legal framework or ownership decision and study the effect of different default structures. For example Halonen (2002) considers a model where the breakdown payoffs allow either one agent to expropriate the entire output if there is single ownership or both agents to expropriate half of the output if there is joint ownership.

\section{CONCLUSION AND FURTHER WORK}

In this paper we have analyzed the dynamic properties of a relational or self-enforcing contract between two risk-neutral agents both of whom undertake a costly investment or action which yields joint benefits. We have shown that there is convergence to a stationary state at which the net surplus is maximized. An optimum contract exhibits a two-phase property. In the first phase (which may or may not occur) there is backloading of the utility of one of the agents. In this phase that agent has zero consumption and will overinvest while the other agent will typically underinvest. In the next phase (which will occur with probability one) there is no overinvestment and after the first period of the this phase there will be either efficient investment, if that is sustainable in that state, or underinvestment by both agents and with both agents constrained.

The analysis presented in the paper is applicable to a wide variety of situations. It will apply to situations of joint ventures where two partners expend individual effort or investment to improve profits. It will apply to a labor market situation where both employer and employee invest in improving the productivity of the job match and it could apply to situations of international trade where trading partners undertake investments to reduce the cost or improve the efficiency of trade. It can also apply with some re-working to a public good model where agents have to decide upon their individual contributions to a public good that benefits both agents.

The model can be extended in a number of directions. An obvious extension is to allow for risk aversion. The assumption that agents cannot borrow introduces some risk aversion but allowing smoothly concave preferences will be an important extension as it will bring together the strand of the literature on self-enforcing contracts which concentrates on risk-sharing with the strand which emphasizes the actions undertaken by agents. It will also broaden the range of applications to include, for example, household behavior. Other extensions could allow for heterogeneous discount rates or to treat the actions as real investments with capital accumulation such as in a model of sovereign debt, or to consider efficient ownership structures when ownership affects the breakdown payoffs.

that this process satisfies our assumptions on the breakdown payoffs, $\phi_{i}\left(a, s_{t}\right)$, all the results go through. 


\section{APPENDIX}

Proof of Lemma 1: By Assumption 1(v) that, holding $a_{j}$ fixed, $y\left(a_{1}, a_{2}, s_{t}\right)$ is strictly concave in $a_{i}$ if increasing, the conditionally efficient actions are uniquely defined. From the continuity and differentiability assumptions $a_{i}^{*}\left(a_{j}, s_{t}\right)$ are continuous functions of $a_{j}$.

Q.E.D.

Proof of Lemma 2: We drop the notational dependence on $s_{t}$ as it is held fixed. Uniqueness of the $a_{i}^{N}\left(a_{j}\right)$ follows from Assumption 3 that $\phi_{i}\left(a_{i}, a_{j}\right)$ is strictly concave in its own action if increasing. Standard results give that these are continuous functions. We have $\partial y\left(a_{i}^{*}\left(a_{j}\right), a_{j}\right) / \partial a_{i} \leq 1$ with equality if $a_{i}^{*}\left(a_{j}\right)>0$. By (1) and the hold-up assumption of Assumption 3, $\partial \phi_{i}(a) / \partial a_{i}<\partial y(a) / \partial a_{i}$ if $\partial y(a) / \partial a_{i}>0$. First consider the case where $a_{i}^{*}\left(a_{j}\right)>0$. Then either $a_{i}^{N}\left(a_{j}\right)=0$ and we're done or if $a_{i}^{N}\left(a_{j}\right)>0$ we have

$$
1=\frac{\partial \phi_{i}\left(a_{i}^{N}\left(a_{j}\right), a_{j}\right)}{\partial a_{i}}=\frac{\partial y\left(a_{i}^{*}\left(a_{j}\right), a_{j}\right)}{\partial a_{i}}>\frac{\partial \phi_{i}\left(a_{i}^{*}\left(a_{j}\right), a_{j}\right)}{\partial a_{i}}
$$

where the first two equalities hold from the first-order conditions for $a_{i}^{N}\left(a_{j}\right)$ and $a_{i}^{*}\left(a_{j}\right)$ and the inequality was argued above. It then follows from the the strict concavity of $\phi_{i}$ in its own argument (Assumption 3) that $a_{i}^{N}\left(a_{j}\right)<a_{i}^{*}\left(a_{j}\right)$. Next suppose that $a_{i}^{*}\left(a_{j}\right)=0$ and contrary to the lemma that $a_{i}^{N}\left(a_{j}\right)>0$. Then again from the first-order and hold-up conditions

$$
1=\frac{\partial \phi_{i}\left(a_{i}^{N}\left(a_{j}\right), a_{j}\right)}{\partial a_{i}}<\frac{\partial y\left(a_{i}^{N}\left(a_{j}\right), a_{j}\right)}{\partial a_{i}}
$$

but

$$
1 \geq \frac{\partial y\left(0, a_{j}\right)}{\partial a_{i}} \geq \frac{\partial y\left(a_{i}^{N}\left(a_{j}\right), a_{j}\right)}{\partial a_{i}}
$$

by the first-order condition for $a_{i}^{*}\left(a_{j}\right)$ and the fact that the marginal product is nonincreasing. Hence we have a contradiction.

Q.E.D.

Proof of Lemma 3: Again we drop the notational dependence on $s_{t}$ as it is inessential for the proof. From Lemma $2, a_{i}^{N}\left(a_{j}\right)$ is a continuous weakly increasing function of $a_{j}$. Then $\phi_{i}\left(a_{i}^{N}\left(a_{j}\right), a_{j}\right)-a_{i}^{N}\left(a_{j}\right)$ and hence $D_{i}\left(a_{j}\right)$ is continuous and differentiable for $a_{i}^{N}\left(a_{j}\right)>0$ (by the theorem of the maximum). As $a_{i}=0$ is feasible $\phi_{i}\left(a_{i}^{N}\left(a_{j}\right), a_{j}\right)-a_{i}^{N}\left(a_{j}\right) \geq \phi_{i}\left(0, a_{j}\right) \geq 0$. Likewise setting $a_{j}=a_{j}^{N E}, \phi_{i}\left(a^{N E}\right)-a_{i}^{N E} \geq 0$. Hence $D_{i}\left(a_{j}\right) \geq 0$. By the envelope theorem $D_{i}^{\prime}\left(a_{j}\right)=\partial \phi_{i}\left(a_{i}^{N}\left(a_{j}\right), a_{j}\right) / \partial a_{j}$ and so by Assumption $3, D_{i}^{\prime}\left(a_{j}\right) \geq 0$. If $\partial y\left(a_{i}^{N}\left(a_{j}\right), a_{j}\right) / \partial a_{j}>0$ then again from Assumption 3 $\partial \phi_{i}\left(a_{i}^{N}\left(a_{j}\right), a_{j}\right) / \partial a_{j}>0$ and so $D_{i}\left(a_{j}\right)$ is strictly increasing in $a_{j}$.

Q.E.D.

Proof of Lemma 4: Consider the strategy for each agent of always playing the breakdown Nash equilibrium actions $a_{i}^{N E}\left(s_{t}\right)$, and demanding the entire output. By definition these are short-run mutual best responses, since for any $a_{i} \geq 0$ if $i$ 's demand is positive the game moves to breakdown (as agent $j$ will demand all output), in which case $a_{i}^{N E}$ followed by demanding $y$ is by definition optimal, and a zero demand will lead to a non-positive payoff, which cannot be greater than the payoff from $a_{i}^{N E}$ 
followed by demanding $y$. Thus a pure-strategy equilibrium exists. Moreover the set of equilibrium payoffs is compact. Briefly, the action-consumption profiles after any history $s^{t}$ must be bounded in equilibrium. To see this note that assumptions on the action sets and the production function mean that actions can be restricted to some compact set $\tilde{A}\left(s_{t}\right) \subseteq \Re_{+}^{2}$ and hence the per-period utility payoffs also belong to a closed and bounded subset $W\left(s_{t}\right) \equiv\left\{w\left(s_{t}\right): w\left(s^{t}\right)+a\left(s^{t}\right) \geq 0, \sum_{i} w_{i}\left(s^{t}\right)+a_{i}\left(s^{t}\right) \leq y\left(a, s_{t}\right)\right.$ and $\left.a \in \tilde{A}\left(s_{t}\right)\right\}$. Thus we can restrict the action-consumption pairs after $s^{t}$ to a compact subset, say $\digamma\left(s^{t}\right) \subset \Re_{+}^{4}$. Hence the product space $\prod_{s^{t}} \digamma\left(s^{t}\right)$ is sequentially compact in the product topology as it is a countable product of compact spaces. Take any convergent sequence of equilibrium payoffs and the associated sequence of self-enforcing contracts. The latter has a convergent sub-sequence that converges pointwise to some limiting contract. Now consider the payoffs after each $s^{t}$ associated with this sequence of contracts. By the dominated convergence theorem the limit must satisfy the self-enforcing constraints (2) since payoffs are continuous functions of contracts in this topology with $\delta<1$, and the constraints are weak inequalities. Thus the limit contract is an equilibrium, and the limiting sequence of equilibrium payoffs has a limit point which corresponds to an equilibrium. It follows the payoff set $V$ is closed and bounded and hence compact subset of $R^{2}$. The existence of optimum contracts then follows by maximizing weighted sums of utilities, with nonnegative weights, over this set.

Q.E.D.

Proof of Lemma 5: The proof proceeds in two parts. The first is to show that one cannot simultaneously have $a_{2}<a_{2}^{N}\left(a_{1}\right)$ and $a_{1}>a_{1}^{N}\left(a_{2}\right)$ or vice-versa. Thus the actions must be either above both reaction functions or below both reaction functions. The next part shows that $a<a^{N E}$ is impossible, ruling out that both are below the reaction functions since the reaction functions are non-decreasing from Lemma 2.

Step 1: Suppose then that at some date $t, a_{2}<a_{2}^{N}\left(a_{1}\right)$ and $a_{1}>a_{1}^{N}\left(a_{2}\right)$. Consider a small increase in $a_{2}$ of $\Delta a_{2}>0$. The consequent increase in output is approximately $\left(\partial y\left(a_{1}, a_{2}\right) / \partial a_{2}\right) \Delta a_{2}$. Change the contract by increasing agent 1's transfer at $t$ by the increase in her deviation payoff, which is to a first order approximation $D_{1}^{\prime}\left(a_{2}\right) \Delta a_{2}=$ $\left(\partial \phi_{1}\left(a_{1}^{N}\left(a_{2}\right), a_{2}\right) / \partial a_{2}\right) \Delta a_{2}$ (by the envelope theorem). The remainder of the extra output (we show this is positive below as agent 2 will be better off) is given to agent 2 . Keep the future unchanged. We now show that these changes meet the constraints and lead to a Pareto-improvement, contrary to the assumed optimality of the contract. First, agent 1 is no worse off (in fact better off, given the hold-up assumption) and by construction her constraint is satisfied. For agent 2, the change in current utility equals the remainder of the output increase less the action cost; to a first-order approximation this is

$$
\Delta w_{2} \simeq\left(\frac{\partial y\left(a_{1}, a_{2}\right)}{\partial a_{2}}-\frac{\partial \phi_{1}\left(a_{1}^{N}\left(a_{2}\right), a_{2}\right)}{\partial a_{2}}-1\right) \Delta a_{2}
$$

Since $a_{2}<a_{2}^{N}\left(a_{1}\right)$ and $\partial^{2} \phi_{2} / \partial a_{2}^{2}<0$ on $\left(a_{2}, a_{2}^{N}\left(a_{1}\right)\right)$ (by Assumption 3, given that $\left.\phi_{2}\left(a_{1}, a_{2}^{N}\left(a_{1}\right)\right)>0\right)$,

$$
\frac{\partial \phi_{2}\left(a_{1}, a_{2}\right)}{\partial a_{2}}>\frac{\partial \phi_{2}\left(a_{1}, a_{2}^{N}\left(a_{1}\right)\right)}{\partial a_{2}}=1
$$


(where the equality follows by virtue of $a_{2}^{N}\left(a_{1}\right)>0$ so there is an interior solution). Since $a_{1}>a_{1}^{N}\left(a_{2}\right)$, and $\partial^{2} \phi_{1} / \partial a_{1} \partial a_{2} \geq 0$, we have

$$
\frac{\partial \phi_{1}\left(a_{1}^{N}\left(a_{2}\right), a_{2}\right)}{\partial a_{2}} \leq \frac{\partial \phi_{1}\left(a_{1}, a_{2}\right)}{\partial a_{2}}
$$

Together (A.2), (A.3) and (1) imply the term in brackets in the right hand side of (A.1) is positive, and thus for $\Delta a_{2}$ small enough, $\Delta w_{2}>0$. Agent 2's constraint is satisfied as $a_{1}$ and hence also $D_{2}\left(a_{1}\right)$ are unchanged, while his utility has risen, so a Paretoimprovement has been demonstrated. A symmetric argument applies if $a_{1}<a_{1}^{N}\left(a_{2}\right)$ and $a_{2}>a_{2}^{N}\left(a_{1}\right)$.

Step 2: Suppose that $\left(a_{1}, a_{2}\right) \leq\left(a_{1}^{N E}, a_{2}^{N E}\right)$ with strict inequality for at least one agent, say agent 2 , and consider replacing the actions with the Nash equilibrium actions $a_{i}^{N E}$ so that output rises from $y\left(a_{1}, a_{2}\right)$ to $y\left(a_{1}^{N E}, a_{2}^{N E}\right)$. We give $\phi_{1}\left(a_{1}^{N E}, a_{2}^{N E}\right)-\phi_{1}\left(a_{1}, a_{2}\right)$ of this increase to agent 1 and the rest to agent 2 (we shall show that utility does not fall, so consumption does not fall and thus the change is feasible). The change in per-period utilities are

$$
\Delta w_{1}=\phi_{1}\left(a_{1}^{N E}, a_{2}^{N E}\right)-\phi_{1}\left(a_{1}, a_{2}\right)-\left(a_{1}^{N E}-a_{1}\right)
$$

and

$$
\begin{aligned}
\Delta w_{2} & =y\left(a_{1}^{N E}, a_{2}^{N E}\right)-y\left(a_{1}, a_{2}\right)-\left(\phi_{1}\left(a_{1}^{N E}, a_{2}^{N E}\right)-\phi_{1}\left(a_{1}, a_{2}\right)\right)-\left(a_{2}^{N E}-a_{2}\right) \\
& \geq \phi_{2}\left(a_{1}^{N E}, a_{2}^{N E}\right)-\phi_{2}\left(a_{1}, a_{2}\right)-\left(a_{2}^{N E}-a_{2}\right)
\end{aligned}
$$

where the inequality follows from integrating equation (1). By definition of $\left(a_{1}^{N E}, a_{2}^{N E}\right)$,

$$
D_{i}\left(a_{j}^{N E}\right)=\phi_{i}\left(a_{1}^{N E}, a_{2}^{N E}\right)-a_{i}^{N E}+\delta \sum_{r \in \mathcal{S}} \pi_{s r} D_{i}\left(a_{j, r}^{N E}, r\right),
$$

for $i \neq j$. Hence for agent 1

$$
\left.D_{1}\left(a_{2}^{N E}\right)-D_{1}\left(a_{2}\right)=\phi_{1}\left(a_{1}^{N E}, a_{2}^{N E}\right)-\phi_{1}\left(a_{1}^{N}\left(a_{2}\right), a_{2}\right)\right)-\left(a_{1}^{N E}-a_{1}^{N}\left(a_{2}\right)\right),
$$

with a similar expression for agent 2. Using (A.4) and (A.5),

$$
\left.\Delta w_{1}-\left(D_{1}\left(a_{2}^{N E}\right)-D_{1}\left(a_{2}\right)\right)=\phi_{1}\left(a_{1}^{N}\left(a_{2}\right), a_{2}\right)\right)-\phi_{1}\left(a_{1}, a_{2}\right)-\left(a_{1}^{N}\left(a_{2}\right)-a_{1}\right) .
$$

By the definition of $a_{1}^{N}\left(a_{2}\right), \phi_{1}\left(a_{1}^{N}\left(a_{2}\right), a_{2}\right)-a_{1}^{N}\left(a_{2}\right) \geq \phi_{1}\left(a_{1}, a_{2}\right)-a_{1}$ for all $a_{1} \geq 0$. Thus $\Delta w_{1}-\left(D_{1}\left(a_{2}^{N E}\right)-D_{1}\left(a_{2}\right)\right) \geq 0$ and the change does not violate the constraint of agent 1 . Likewise for agent 2 . To obtain a contradiction, it remains to show that $\Delta w_{1}, \Delta w_{2} \geq 0$, with at least one strict inequality. As $D_{i}\left(a_{j}^{N E}\right)-D_{i}\left(a_{j}\right) \geq 0$ by $D_{i}(\cdot)$ non-decreasing, it follows from (A.6) that $\Delta w_{1}, \Delta w_{2} \geq 0$. Moreover as $0 \leq a_{2}<a_{2}^{N E}$, there is an interior solution for $a_{2}^{N}\left(a_{1}^{N E}\right)$ so that $\partial \phi_{2}\left(a_{1}^{N E}, a_{2}^{N E}\right) / \partial a_{2}=1$ and then from Assumption $3 \partial y\left(a_{1}^{N E}, a_{2}^{N E}\right) / \partial a_{2}>0$ and output increases as $a_{2}$ is increased. Thus by from Lemma $3, D_{1}^{\prime}\left(a_{2}^{N E}\right)>0$ and therefore $D_{1}\left(a_{2}^{N E}\right)-D_{1}\left(a_{2}\right)>0$, and hence $\Delta w_{1}>0$ as required.

Q.E.D. 
Proof of Lemma 6: (i) If $a_{j}<a_{j}^{*}\left(a_{i}\right)$ then raising $a_{j}$ by $\Delta a_{j}$ sufficiently small will not violate agent $i$ ' self-enforcing constraint as $D_{i}(\cdot)$ is continuous, and will produce more output. Giving this extra output to agent $j$, the change in his utility is $\Delta w_{j} \simeq$ $\left(\left(\partial y\left(a_{1}, a_{2}\right) / \partial a_{j}\right)-1\right) \Delta a_{j}$. Since $a_{j}<a_{j}^{*}\left(a_{i}\right),\left(\partial y\left(a_{1}, a_{2}\right) / \partial a_{j}\right)>1$ and hence utility is improved without violating any constraint agent $j$ 's deviation utility is unchanged so his constraint is relaxed. This contradicts the assumed optimality of the initial contract. (ii) Suppose for agent 1 say, $a_{1}>a_{1}^{*}\left(a_{2}\right)$ and $c_{1}>0$. Consider cutting $a_{1}$ and $c_{1}$ by the same small amount, that is $\Delta c_{1}=\Delta a_{1}<0$. Then $\Delta w_{1}=\Delta c_{1}-\Delta a_{1}=0$. Transfer this cut in consumption to agent 2 and reduce his consumption by the reduction in output. That is $\Delta c_{2} \simeq-\Delta c_{1}+\left(\partial y\left(a_{1}, a_{2}\right) / \partial a_{1}\right) \Delta a_{1}=-\left(1-\left(\partial y\left(a_{1}, a_{2}\right) / \partial a_{1}\right)\right) \Delta a_{1}$. As $\left(\partial y\left(a_{1}, a_{2}\right) / \partial a_{1}\right)<1$ by $a_{1}>a_{1}^{*}\left(a_{2}\right), \Delta w_{2}=\Delta c_{2}>0$ showing that a Paretoimprovement can be found. No constraints are violated as agent 1's deviation payoff is unchanged ( $a_{2}$ is held constant) and agent 2 's deviation payoff falls with $a_{1}$. Q.E.D.

Proof of Lemma 7: By definition $a_{2}^{*}\left(a_{1}\right)=\arg \max _{a_{2}^{\prime}}\left\{y\left(a_{1}, a_{2}^{\prime}\right)-a_{2}^{\prime}\right\}$ and by diminishing marginal product $\partial^{2} y\left(a_{1}, a_{2}\right) / \partial a_{2}^{2} \leq 0$, so that $y\left(a_{1}, a_{2}^{\prime}\right)-a_{2}^{\prime}$ is weakly increasing in $a_{2}^{\prime}$ for $a_{2}^{N}\left(a_{1}\right) \leq a_{2}^{\prime} \leq a_{2}^{*}\left(a_{1}\right)$. In particular, since $a_{2}^{N}\left(a_{1}\right) \leq a_{2} \leq a_{2}^{*}\left(a_{1}\right)$,

$$
y\left(a_{1}, a_{2}\right)-a_{2} \geq y\left(a_{1}, a_{2}^{N}\left(a_{1}\right)\right)-a_{2}^{N}\left(a_{1}\right) .
$$

Since in breakdown agent 2 receives at most all output,

$$
\begin{aligned}
D_{2}\left(a_{1}\right) & \equiv \phi_{2}\left(a_{1}, a_{2}^{N}\left(a_{1}\right)\right)-a_{2}^{N}\left(a_{1}\right)+\delta \sum_{r} \pi_{s r} D_{2}\left(a_{1, r}^{N E}, r\right) \\
& \leq y\left(a_{1}, a_{2}^{N}\left(a_{1}\right)\right)-a_{2}^{N}\left(a_{1}\right)+\delta \sum_{r} \pi_{s r} D_{2}\left(a_{1, r}^{N E}, r\right) \\
& \leq y\left(a_{1}, a_{2}\right)-a_{2}+\delta \sum_{r} \pi_{s r} D_{2}\left(a_{1, r}^{N E}, r\right),
\end{aligned}
$$

where the second inequality follows from (A.7), and since $V_{2, r} \geq D_{2}\left(a_{1, r}^{N E}, r\right)$, all $r \in S$, this implies (3) is satisfied. Next suppose $a_{2}>0$. If $a_{2}^{N}\left(a_{1}\right)=0$, then it follows from $a_{2}^{*}\left(a_{1}\right) \geq a_{2}>0$ that $a_{2}^{*}\left(a_{1}\right)>a_{2}^{N}\left(a_{1}\right)$ and hence from Assumption $1, \partial y\left(a_{1}, a_{2}\right) / \partial a_{2}>0$ and thus $\partial^{2} y\left(a_{1}, a_{2}\right) /\left(\partial a_{2}\right)^{2}<0$ on $\left(0, a_{2}^{*}\left(a_{1}\right)\right)$. Thus (A.7) holds strictly. If $a_{2}^{N}\left(a_{1}\right)>$ 0 then $y\left(a_{1}, a_{2}^{N}\left(a_{1}\right)\right)>0$ and (A.8) is strict by Assumption 3 which implies that $\phi_{2}\left(a_{1}, a_{2}^{N}\left(a_{1}\right)\right)<y\left(a_{1}, a_{2}^{N}\left(a_{1}\right)\right)$; so in either case (3) holds strictly.

Q.E.D.

Proof of Theorem 1: (i) Assume by contradiction that agent 1 is unconstrained and $a_{1}^{\tilde{t}}<a_{1}^{*}\left(a_{2}^{\tilde{t}}\right)$, but that $c_{2}^{t}>0$ after $s^{t}$ for some $t<\tilde{t}$ (where $s^{t}$ is composed of the first $t+1$ components of $\left.s^{\tilde{t}}\right)$. Assume w.l.o.g. that $c_{2}^{t^{\prime}}=0$ for $t<t^{\prime}<\tilde{t}$ (i.e., choose $t$ so that this is satisfied on $s^{\tilde{t}}$ ). We shall change the contract at dates $t$ and $\tilde{t}$ (only), and demonstrate an improvement. Consider a small increase in $a_{1}^{\tilde{t}}$, $\Delta a_{1}^{\tilde{t}}>0$, and let $\tilde{\Delta}\left(\simeq D_{2}^{\prime}\left(a_{1}^{\tilde{t}}\right) \Delta a_{1}^{\tilde{t}}\right)$ be the resulting increase in agent 2 's deviation payoff. To preserve agent 2 's self-enforcing constraint, transfer $\tilde{\Delta}$ from agent 1 at date $\tilde{t}$ (i.e., $\Delta c_{2}^{\tilde{t}}=\tilde{\Delta}$ ), and allocate the remaining incremental output to agent 1 , $\Delta c_{1}^{\tilde{t}} \simeq\left(\partial y\left(a_{1}^{\tilde{t}}, a_{2}^{\tilde{t}}\right) / \partial a_{1}\right) \Delta a_{1}^{\tilde{t}}-\tilde{\Delta}$. This is feasible since $\Delta c_{1}^{\tilde{t}} \geq 0$. The argument for this is similar to that in the proof of Lemma 5 (Step 1). Inequality (1) in Assumption 3, along with $\partial \phi_{2}(a) / \partial a_{1} \geq 0$ implies $\partial \phi_{2}(a) / \partial a_{1} \leq \partial y(a) / \partial a_{1}$. Then using the envelope 
theorem, $D_{2}^{\prime}\left(a_{1}\right)=\partial \phi_{2}\left(a_{1}, a_{2}^{N}\left(a_{1}\right)\right) / \partial a_{1} \leq \partial \phi_{2}\left(a_{1}, a_{2}\right) / \partial a_{1}$ since $a_{2} \geq a_{2}^{N}\left(a_{1}\right)$ (by Lemma 5) and $\partial^{2} \phi_{2} / \partial a_{1} \partial a_{2} \geq 0$. Thus $D_{2}^{\prime}\left(a_{1}\right) \leq \partial y(a) / \partial a_{1}$ and the result follows from integrating between $a_{1}^{\tilde{t}}$ and $a_{1}^{\tilde{t}}+\Delta a_{1}^{\tilde{t}}$. Agent 1's self-enforcing constraint holds as it was slack initially. Thus both self-enforcing constraints hold at $\tilde{t}$. At the earlier date $c_{2}^{t}>0$, so the increase in agent 1's effort can be compensated at $t$, and the increase in surplus must imply a Pareto-improvement at $t$. Specifically: cut agent 2 's consumption at date $t$ so that $\Delta c_{2}^{t}=-\delta^{\tilde{t}-t} \tilde{\pi} \tilde{\Delta}<0$ where $\tilde{\pi}>0$ is the probability of reaching $s^{\tilde{t}}$ from $s^{t}$. This consumption is given to agent 1 so $\Delta c_{1}^{t}=-\Delta c_{2}^{t}>0$. Thus the change in discounted utility for agent 1 at date $t$ is

$$
\begin{aligned}
\Delta V_{1}\left(s^{t}\right) & =\delta^{\tilde{t}-t} \tilde{\pi} \tilde{\Delta}+\delta^{\tilde{t}-t} \tilde{\pi}\left(\Delta c_{1}^{\tilde{t}}-\Delta a_{1}^{\tilde{t}}\right) \\
& \simeq \delta^{\tilde{t}-t} \tilde{\pi} \tilde{\Delta}+\delta^{\tilde{t}-t} \tilde{\pi}\left(\left(\frac{\partial y\left(a_{1}^{\tilde{t}}, a_{2}^{\tilde{t}}\right)}{\partial a_{1}}-1\right) \Delta a_{1}^{\tilde{t}}-\tilde{\Delta}\right) \\
& =\delta^{\tilde{t}-t} \tilde{\pi}\left(\frac{\partial y\left(a_{1}^{\tilde{t}}, a_{2}^{\tilde{t}}\right)}{\partial a_{1}}-1\right) \Delta a_{1}^{\tilde{t}}
\end{aligned}
$$

which is positive as $a_{1}^{\tilde{t}}$ is under-efficient by assumption and so $\partial y\left(a_{1}^{\tilde{t}}, a_{2}^{\tilde{t}}\right) / \partial a_{1}>1$. Likewise the change for agent 2 is $\Delta V_{2}\left(s^{t}\right)=-\delta^{\tilde{t}-t} \tilde{\pi} \tilde{\Delta}+\delta^{\tilde{t}-t} \tilde{\pi} \tilde{\Delta}=0$. Thus the period $t$ constraints hold as actions are unchanged, and there is a Pareto-improvement. It remains to check the constraints at periods $t^{\prime}$ for $t<t^{\prime}<\tilde{t}$ : agent 2 's future utility (at $\tilde{t})$ has increased so his constraints still hold. However agent 1's utility at $\tilde{t}$ may have fallen, decreasing her payoff at $t^{\prime}$. Nevertheless, agent 1 gets all consumption so $c_{1}^{t^{\prime}}>0$ and hence by Lemma 6(ii) $a_{1}^{t^{\prime}} \leq a_{1}^{*}\left(a_{2}^{t^{\prime}}\right)$, unless $y\left(a^{t^{\prime}}\right)=0$, in which case $a_{1}^{t^{\prime}}=0$, so again $a_{1}^{t^{\prime}} \leq a_{1}^{*}\left(a_{2}^{t^{\prime}}\right)$. Also by Lemma $5 a_{1}^{t^{\prime}} \geq a_{1}^{N}\left(a_{2}^{t^{\prime}}\right)$. Likewise by Lemma $5 a_{2}^{\tilde{t}} \geq a_{2}^{N E}$, and we know that agent 1 's constraint holds at $\tilde{t}$, so $\tilde{V}_{1}\left(s^{\tilde{t}}\right) \geq D_{1}\left(a_{2}^{\tilde{t}}\right) \geq D_{1}\left(a_{2}^{N E}\right)$ as $D_{1}(\cdot)$ non-decreasing, where $\tilde{V}_{1}\left(s^{\tilde{t}}\right) \equiv V_{1}\left(s^{\tilde{t}}\right)+\Delta c_{1}^{\tilde{t}}-\Delta a_{1}^{\tilde{t}}$ is agent 1 's new utility. In the other states at $\tilde{t}$, the corresponding inequality holds by equilibrium (nothing has been changed), so continuation utilities after $t^{\prime}=\tilde{t}-1$ satisfy $V_{1, r} \geq D_{1}\left(a_{2, r}^{N E}, r\right)$, for all $r \in S$. Lemma 7 can thus be applied to ensure agent 1's constraints hold at $\tilde{t}-1$. Working backwards, the same holds for all $t^{\prime}>t$. As all the constraints are met a Pareto-improvement has been found.

(ii) We now prove the second part of the theorem. Assume by contradiction that $a_{2}^{\tilde{t}}>a_{2}^{*}\left(a_{1}^{\tilde{t}}\right)$ but that $c_{2}^{t}>0$ after $s^{t}$ for some $t<\tilde{t}$ (i.e., earlier on the same history). Assume w.l.o.g. that $c_{2}^{t^{\prime}}=0$ for $t<t^{\prime}<\tilde{t}$ (we can choose $t$ so that this is satisfied on $\left.s^{\tilde{t}}\right)$. We shall change the contract at dates $t$ and $\tilde{t}$ (only), and demonstrate a Paretoimprovement. By Lemma 6 (ii) as $a_{2}^{\tilde{t}}>a_{2}^{*}\left(a_{1}^{\tilde{t}}\right)$ we must have $c_{2}^{\tilde{t}}=0$ and so $c_{1}^{\tilde{t}}=y\left(a_{1}^{\tilde{t}}, a_{2}^{\tilde{t}}\right)$. Now consider a small change in $a_{2}^{\tilde{t}}$ of $\Delta a_{2}^{\tilde{t}}<0$, but continue allocating all output to agent 1 . If $a_{1}^{\tilde{t}}>0$ then since $c_{1}^{\tilde{t}}=y\left(a_{1}^{\tilde{t}}, a_{2}^{\tilde{t}}\right)>0$ and thus $a_{1}^{\tilde{t}} \leq a_{1}^{*}\left(a_{2}^{\tilde{t}}\right)$ by Lemma 6(ii) (and $a_{1}^{\tilde{t}} \geq a_{1}^{N}\left(a_{2}^{\tilde{t}}\right)$ by Lemma 5 ), agent 1 is initially unconstrained by Lemma 7 and a small cut in the consumption of agent 1 will not violate his constraint. If on the other hand $a_{1}^{\tilde{t}}=0$, then after the change we have, trivially, $a_{1}^{\tilde{t}} \leq a_{1}^{*}\left(a_{2}^{\tilde{t}}+\Delta a_{2}^{\tilde{t}}\right)$, while $a_{1}^{N}\left(a_{2}^{\tilde{t}}+\Delta a_{2}^{\tilde{t}}\right) \leq a_{1}^{N}\left(a_{2}^{\tilde{t}}\right) \leq a_{1}^{\tilde{t}}(=0)$ as $a_{1}^{N}(\cdot)$ is non-decreasing. So Lemma 7 applies and again agent 1's constraint must hold. For agent 2 since $a_{1}^{\tilde{t}}$ is unchanged and $w_{2}^{\tilde{t}}$ is increased (the cut in effort implies $\Delta w_{2}^{\tilde{t}}=-\Delta a_{2}^{\tilde{t}}>0$ ), his self-enforcing constraint is satisfied at $\tilde{t}$. Thus both self-enforcing constraints hold at $\tilde{t}$. Agent 1 is getting all 
consumption and so satisfies the self-enforcing constraint at all intervening dates $t^{\prime}$, $t<t^{\prime}<\tilde{t}$, repeating the argument from part (i) of the proof, while agent 2 is better off due to the improvement at $\tilde{t}$, so his constraints are not violated (as deviation payoffs are unchanged at $t^{\prime}$ ). The increase in surplus at $\tilde{t}$ allows for a Pareto-improvement at $t$ : To compensate agent 1 at date $t$ for any decreased consumption at date $\tilde{t}, \Delta c_{1}^{\tilde{t}}<0$, let $\Delta c_{1}^{t}=-\delta^{(\tilde{t}-t)} \tilde{\pi} \Delta c_{2}^{\tilde{t}}>0$, where we denote by $\tilde{\pi}>0$ the probability of reaching $s^{\tilde{t}}$ at date $\tilde{t}$ starting from $t$ earlier on the same history. We take this increase from agent 2, so $\Delta c_{2}^{t}=\delta^{(\tilde{t}-t)} \tilde{\pi} \Delta c_{1}^{\tilde{t}}$ and since $\Delta c_{1}^{\tilde{t}} \simeq\left(\partial y\left(a_{1}^{\tilde{t}}, a_{2}^{\tilde{t}}\right) / \partial a_{2}\right) \Delta a_{2}^{\tilde{t}}$ we have that the change in discounted utility for agent 2 is

$$
\Delta V_{2}\left(s^{t}\right) \simeq \delta^{(\tilde{t}-t)} \tilde{\pi}\left(\left(\partial y\left(a_{1}^{\tilde{t}}, a_{2}^{\tilde{t}}\right) / \partial a_{2}\right)-1\right) \Delta a_{2}^{\tilde{t}}>0
$$

since $\partial y\left(a_{1}^{\tilde{t}}, a_{2}^{\tilde{t}}\right) / \partial a_{2}<1\left(\right.$ as $\left.a_{2}^{\tilde{t}}>a_{2}^{*}\left(a_{1}^{\tilde{t}}\right)\right)$ and $\Delta a_{2}^{\tilde{t}}<0$. This increase at date $t$ implies that both self-enforcing constraints hold at $t$ (actions are unchanged, so deviation payoffs are unchanged) and hence the original contract was not optimum.

Q.E.D.

Proof of Lemma 8: To show the connection between a joint utility maximizing and current surplus maximizing self-enforcing contract, it will be useful to define an intermediate case where the action profile maximizes current surplus for a given set of continuation utilities. We say that the action vector $a$ is myopic surplus maximizing in state $s$ for continuation utilities $\left(V_{1, r}, V_{2, r}\right)_{r \in \mathcal{S}}$ if there is an associated consumption vector $c$ such that $(a, c) \in \arg \max _{(a, c) \in R_{+}^{4}}\left(y\left(a_{1}, a_{2}, s\right)-a_{1}-a_{2}\right)$ s.t. $c_{i}-a_{i}+\delta \sum_{r \in \mathcal{S}} \pi_{s r} V_{i, r} \geq D_{i}\left(a_{j}, s\right)$, and $c_{1}+c_{2} \leq y\left(a_{1}, a_{2}\right)$ for $i=1,2, j \neq i$. (Myopic surplus maximizing actions are not necessarily optimum since they take the continuation utilities as given and do not take into account the trade-off between actions today and actions in the future.)

Step 1: We show that if $\tilde{a}$ is myopic surplus maximizing for some $\left(V_{1, r}, V_{2, r}\right)_{r \in \mathcal{S}}$ with $V_{i, r} \geq D_{i}\left(a_{j, r}^{N E}, r\right)$ all $r \in \mathcal{S}, i, j=1,2, j \neq i$, then

$$
y\left(\tilde{a}_{1}, \tilde{a}_{2}, s\right)-\tilde{a}_{i}+\delta \sum_{r \in \mathcal{S}} \pi_{s r} D_{i}\left(a_{j, r}^{N E}, r\right) \geq D_{i}\left(\tilde{a}_{j}, s\right),
$$

for $i, j=1,2, j \neq i$; i.e., giving all output to agent $i$ implies that the current selfenforcing constraint continues to hold for agent $i$ even if the continuation utilities are replaced with the deviation payoffs. By adapting the proof of Lemma 5 it is easy to check that $\tilde{a}_{i} \geq a_{i}^{N}\left(\tilde{a}_{j}\right)$ for $i, j=1,2, j \neq i$. Next, suppose $\tilde{a}_{i}>a_{i}^{*}\left(\tilde{a}_{j}\right)$. We shall establish a contradiction. If $c_{i}>0$ then lowering both $a_{i}$ and $c_{i}$ by an equal small amount is feasible and does not violate either constraint: agent $i$ is no worse off, and $j \neq i$ is strictly better off (by the net surplus increase), contrary to assumption. Thus $\tilde{a}_{i}>a_{i}^{*}\left(\tilde{a}_{j}\right)$ and $c_{i}>0$ is impossible. If $c_{i}=0$ and hence $c_{j}=y\left(\tilde{a}_{1}, \tilde{a}_{2}\right)$ then lowering $a_{i}$ will lower the consumption of agent $j$ and hence might violate agent $j$ 's self-enforcing constraint. But $\tilde{a}_{i}>a_{i}^{*}\left(\tilde{a}_{j}\right)$ implies $y\left(\tilde{a}_{1}, \tilde{a}_{2}\right)>0$ so $c_{j}>0$ and thus $\tilde{a}_{j} \leq a_{j}^{*}\left(\tilde{a}_{i}\right)$ by the above argument. Suppose that $a_{i}$ is reduced to $a_{i}^{*}\left(\tilde{a}_{j}\right)$ with agent $j$ still receiving all the output. Clearly the surplus has increased, and agent $i$ 's utility has risen while $D_{i}\left(\tilde{a}_{j}\right)$ is unchanged, so that agent $i$ 's self-enforcing constraint is still satisfied. There are two cases to consider.

(i) If $\tilde{a}_{j} \leq a_{j}^{*}\left(a_{i}^{*}\left(\tilde{a}_{j}\right)\right)$ then leave $a_{j}$ unchanged at $\tilde{a}_{j}$. Thus, as $a_{j}^{N}$ can only fall with the 
cut in $a_{i}, a_{j}^{N}\left(a_{i}^{*}\left(\tilde{a}_{j}\right)\right) \leq \tilde{a}_{j} \leq a_{j}^{*}\left(a_{i}^{*}\left(\tilde{a}_{j}\right)\right)$. All the conditions of Lemma 7 are satisfied at $\left(a_{i}^{*}\left(\tilde{a}_{j}\right), \tilde{a}_{j}\right)$, so the current self-enforcing constraint for agent $j$ holds:

$$
y\left(a_{i}^{*}\left(\tilde{a}_{j}\right), \tilde{a}_{j}\right)-\tilde{a}_{j}+\delta \sum_{r \in \mathcal{S}} \pi_{s r} V_{j, r} \geq D_{j}\left(a_{i}^{*}\left(\tilde{a}_{j}\right)\right) .
$$

(ii) As $a_{i}$ is reduced $a_{j}^{*}\left(a_{i}\right)$ may have fallen below $\tilde{a}_{j}$. If this is the case, cut $a_{j}$ to $a_{j}^{*}\left(a_{i}^{*}\left(\tilde{a}_{j}\right)\right)$. Repeating the argument just given, agent $j$ 's constraint will be satisfied at $\left(a_{i}^{*}\left(\tilde{a}_{j}\right), a_{j}^{*}\left(a_{i}^{*}\left(\tilde{a}_{j}\right)\right)\right)$, while the cut in $a_{j}$ cannot lead to a violation in agent $i$ 's constraint. So again the changed contract satisfies the self-enforcing constraints.

In both cases (i) and (ii) the reduction in overinvestment leads to an increase in current surplus, contrary to the assumption that $\tilde{a}$ was myopic surplus maximizing. We conclude that $\tilde{a}_{i}>a_{i}^{*}\left(\tilde{a}_{j}\right)$ is impossible. Thus $a_{i}^{*}\left(\tilde{a}_{j}\right) \geq \tilde{a}_{i} \geq a_{i}^{N}\left(\tilde{a}_{j}\right)$. Thus Lemma 7 can again be appealed to, at $\tilde{a}$ with continuation utilities set equal to $D_{i}\left(a_{j, r}^{N E}, r\right)$, establishing (A.9). Step 2: Take any myopic surplus maximizing action $a$ for some $\left(V_{1, r}, V_{2, r}\right)_{r \in \mathcal{S}}$; we shall show that given any alternative continuation utilities $\left(\hat{V}_{1, r}, \hat{V}_{2, r}\right)_{r \in \mathcal{S}}$ satisfying, for all $r \in \mathcal{S}, \hat{V}_{1, r}+\hat{V}_{2, r} \geq V_{1, r}+V_{2, r}, \hat{V}_{i, r} \geq D_{i}\left(a_{j, r}^{N E}, r\right)$, there is a division of $y\left(a_{1}, a_{2}\right)$ such that the self-enforcing constraints are satisfied with the same action $a$. We need to show that both self-enforcing constraints can still hold with some output division $\left(\hat{c}_{1}, \hat{c}_{2}\right) \geq 0$, where $\hat{c}_{1}+\hat{c}_{2}=y\left(a_{1}, a_{2}\right)$, i.e.,

$$
\hat{c}_{i}-a_{i}+\delta \sum_{r} \pi_{s r} \hat{V}_{i, r} \geq D_{i}\left(a_{j}\right)
$$

for $i, j=1,2, j \neq i$. By assumption they hold in the equilibrium supporting $a$ :

$$
c_{i}-a_{i}+\delta \sum_{r} \pi_{s r} V_{i, r} \geq D_{i}\left(a_{j}\right)
$$

for $i, j=1,2 j \neq i$. Let $i=1$. If (A.10) holds at $\hat{c}_{1}=0$, then setting $\hat{c}_{2}=y\left(a_{1}, a_{2}\right)$ guarantees that (A.10) holds also for agent 2 by Step 1 (because $\hat{V}_{i, r} \geq D_{i}\left(a_{j, r}^{N E}, r\right)$ all $r$ ). Otherwise choose $\hat{c}_{1}$ such that (A.10) holds with equality for $i=1$; by continuity this is possible as (A.10) holds at $\hat{c}_{1}=y\left(a_{1}, a_{2}\right)$ using Step 1 again. Suppose that (A.10) is then violated for $i=2$. Summing the left hand side of (A.10) over $i$ thus implies

$$
y\left(a_{1}, a_{2}\right)-\sum_{i} a_{i}+\delta \sum_{r} \pi_{s r} \sum_{i} \hat{V}_{i, r}<\sum_{i} D_{i}\left(a_{j}\right) .
$$

But summing (A.11) over $i$ implies that

$$
y\left(a_{1}, a_{2}\right)-\sum_{i} a_{i}+\delta \sum_{r} \pi_{s r} \sum_{i} V_{i, r} \geq \sum_{i} D_{i}\left(a_{j}\right)
$$

and since the left hand side is smaller than the left hand side of (A.12) by $\hat{V}_{1, r}+\hat{V}_{2, r} \geq$ $V_{1, r}+V_{2, r}$, there is a contradiction. Hence we conclude that there is a division of $y\left(a_{1}, a_{2}\right)$ such that (A.10) holds for both agents.

Step 3: Consider the following putative equilibrium. At date $t$, in state $s_{t}$, set $a$ to a current joint surplus maximizing action (i.e., compatible with equilibrium) in this state. Adapting earlier arguments, the maximizing actions exist. Let $\left(V_{1, r}, V_{2, r}\right)_{r \in \mathcal{S}}$ be the 
corresponding continuation utilities from $t+1$ in this equilibrium. At time $t+1$, in any state $r$, follow an equilibrium that maximizes joint utility from that point onwards, yielding utilities we denote by $\left(\hat{V}_{1, r}, \hat{V}_{2, r}\right)_{r \in \mathcal{S}}$. Clearly $a$ is myopic surplus maximizing relative to $\left(V_{1, r}, V_{2, r}\right)_{r \in \mathcal{S}}$, so by Step 2 there is a split of $y\left(a_{1}, a_{2}\right)$ which sustains this as an equilibrium from $t$ on when $\left(V_{1, r}, V_{2, r}\right)_{r \in \mathcal{S}}$ is replaced by $\left(\hat{V}_{1, r}, \hat{V}_{2, r}\right)_{r \in \mathcal{S}}$ since the latter have a maximal sum in each state and $\hat{V}_{i, r} \geq D_{i}\left(a_{j, r}^{N E}, r\right), i=1,2$. Note that this must provide maximal joint utility from $t$ since current joint surplus is maximized at $t$, and joint utilities are maximal from $t+1$. Consequently starting from any state $s_{t}$, a joint utility maximizing equilibrium must involve a current joint net output maximizing action compatible with equilibrium in state $s_{t}$, for if it did not, replacing it by the equilibrium just constructed would lead to a higher utility sum. At $t+1$, since the utility sum is maximal in each state $r$, repeating the above argument again confirms that net output is maximal for state $r$. So a joint utility maximizing equilibrium must involve a joint net output maximizing action compatible with equilibrium in every state and date.

Q.E.D.

Proof of Lemma 9: By assumption that both constraints bind we have

$$
c_{i}-a_{i}+\delta \sum_{r} \pi_{s r} V_{i, r}=D_{i}\left(a_{j}\right)
$$

for $i, j=1,2, i \neq j$. Suppose, to the contrary of the claim, that the pair $\left(V_{1, r^{\prime}}, V_{2, r^{\prime}}\right)$ does not maximize joint utility in at least one successor state $r^{\prime}$. We can change the contract as follows. Replace $\left(V_{1, r^{\prime}}, V_{2, r^{\prime}}\right)$ by $\left(\hat{V}_{1, r^{\prime}}, \hat{V}_{2, r^{\prime}}\right) \in V_{r^{\prime}}$ such that $\hat{V}_{1, r^{\prime}}+\hat{V}_{2, r^{\prime}}>V_{1, r^{\prime}}+V_{2, r^{\prime}}$ (and recall that we must have $\left.\hat{V}_{i, r^{\prime}} \geq D_{i}\left(a_{j, r^{\prime}}^{N E}, r^{\prime}\right), i, j=1,2, j \neq i\right)$, and choose a division $\hat{c}$ of the current output $y\left(a_{1}, a_{2}\right)$ (i.e., holding $a$ constant) such that

$$
\hat{c}_{i}-a_{i}+\delta \sum_{r \neq r^{\prime}} \pi_{s r} V_{i, r}+\delta \pi_{s r^{\prime}} \hat{V}_{i, r^{\prime}} \geq D_{i}\left(a_{j}\right)
$$

for $i, j=1,2, j \neq i$, with a strict inequality for at least one $i$. This is possible by the fact that if $\hat{c}_{i}=y\left(a_{1}, a_{2}\right)$ then, as $a_{i} \geq a_{i}^{N}\left(\tilde{a}_{j}\right)$ by Lemma $5, a_{i} \leq a_{i}^{*}\left(a_{j}\right)$ by hypothesis, and $V_{i, r} \geq D_{i}\left(a_{j, r}^{N E}, r\right), r \neq r^{\prime}, \hat{V}_{i, r^{\prime}} \geq D_{i}\left(a_{j, r^{\prime}}^{N E}, r^{\prime}\right)$, the self-enforcing constraint for agent $i$ must be satisfied (Lemma 7). The argument then follows the proof of Lemma 8, step 2; however the increase in aggregate utility implies the constraint (A.13) is strict for one agent. This is a Pareto-improvement, so the original contract could not have been optimal.

Q.E.D.

Proof of Theorem 2: Suppose that $\Lambda(\mathcal{V}) \neq(0,0)$; otherwise the proposition is trivial. ${ }^{47}$

(i) Consider first the case where there exists $\left(V_{1}, V_{2}\right) \in \Lambda(\mathcal{V})$ with $V_{1}, V_{2}>0$. Thus in any optimum, with payoffs $\left(\hat{V}_{1}, \hat{V}_{2}\right)$, either $\hat{V}_{1} \geq V_{1}>0$, or $\hat{V}_{2} \geq V_{2}>0$. We deal first with the former case. In this optimum, choose $t^{\prime}>0$ so that $\delta^{t^{\prime}} \bar{w} /(1-\delta)<V_{1} / 2$, where $\bar{w}$ is an upper bound on equilibrium per-period payoffs (that per-period payoffs are bounded follows from Assumption 1(vi)). Then since the initial expected discounted utility is $\hat{V}_{1} \geq V_{1}$ it follows that even if utility for agent 1 is as high as possible after

\footnotetext{
${ }^{47}$ If $\Lambda(\mathcal{V})=(0,0)$ current surplus maximizing actions lead to a surplus of 0 at all $s$, so $T=0$ as in equilibrium at each $t \geq 0$ surplus must be zero.
} 
date $t^{\prime}$ there must be some history $s^{t}, t<t^{\prime}$, which occurs with positive probability, where $c_{1}^{t} \geq \mu_{1} \equiv V_{1} / 2 t^{\prime}>0$, otherwise the initial utility $V_{1}$ could not be achieved. For convenience we let $s_{t}=r$. Since $c_{1}^{t} \geq \mu_{1}$ we have $y\left(a_{1}^{t}, a_{2}^{t}, r\right) \geq \mu_{1}>0$. We next show that this in turn implies a positive probability that $c_{2}$ must be greater than a positive bound within a fixed amount of time after $s^{t}$ occurs. (a) If $y(\cdot, r)$ is such that only agent 2 can contribute to output, we have $a_{2}^{t} \geq \underline{a}_{2}$ where $y\left(0, \underline{a}_{2}, r\right)=\mu_{1}$. Let $\tilde{C}_{i}\left(s^{t}\right)$ denote expected discounted consumption of agent $i$ to date $t$, conditional on $s^{t}$; the negative utility from $a_{2}^{t}>0$ must be compensated (since $D_{2}\left(a_{1}^{t}, r\right) \geq 0$ ) by positive consumption now or in the future, that is $\tilde{C}_{2}\left(s^{t}\right) \geq \underline{a}_{2}$. (b) If, on the other hand, only agent 1 can contribute, $a_{1}^{t} \geq \underline{a}_{1}$ where $y\left(\underline{a}_{1}, 0, r\right)=\mu_{1}$. Then $D_{2}\left(a_{1}^{t}, r\right) \geq D_{2}\left(\underline{a}_{1}, r\right)>0$, where the strict inequality follows from the hold-up assumption ( $y$ must be increasing in $\left.a_{1}\right)$. It follows that $V\left(s^{t}\right) \geq \tilde{C}_{2}\left(s^{t}\right) \geq D_{2}\left(\underline{a}_{1}, r\right)$ to maintain agent 2 's self-enforcing constraint. (c) Finally, if both can contribute, there are two further cases to consider. Define $\bar{a}_{1}$ to be an upper bound on $a_{1}$ (recall this exists by Assumption 1(vi)). If $y\left(\bar{a}_{1}, 0, r\right)<\mu_{1}$ then define $\underline{a}_{2}>0$ to be such that $y\left(\bar{a}_{1}, \underline{a}_{2}, r\right)=\mu_{1}$ (this exists by the hypothesis that $y\left(a_{1}^{t}, a_{2}^{t}, r\right) \geq \mu_{1}$, by $y$ non-decreasing and by continuity); clearly (by monotonicity) $a_{2}^{t} \geq \underline{a}_{2}$, and so $\tilde{C}_{2}\left(s^{t}\right) \geq \underline{a}_{2}$, as in (a) above. If on the other hand $y\left(\bar{a}_{1}, 0, r\right) \geq \mu_{1}$, define $\tilde{a}_{1}$ so that $y\left(\tilde{a}_{1}, 0, r\right)=\mu_{1}$. By the hold-up assumption (Assumption 3) and because $y$ is increasing in $a_{1}$ when $a_{2}=0, \phi_{2}\left(\tilde{a}_{1}, 0, r\right)>0$. Consequently $D_{2}\left(\tilde{a}_{1}, r\right) \geq \phi_{2}\left(\tilde{a}_{1}, 0, r\right)>0$. By continuity there exists $\left(\underline{a}_{1}, \underline{a}_{2}\right)$ with $\underline{a}_{2}>0$ such that $y\left(\underline{a}_{1}, \underline{a}_{2}, r\right)=\mu_{1}$, with $D_{2}\left(\underline{a}_{1}, r\right) \geq D_{2}\left(\tilde{a}_{1}, r\right) / 2$. Clearly, either $a_{1}^{t} \geq \underline{a}_{1}>0$ or $a_{2}^{t} \geq \underline{a}_{2}>0$. In the case where $a_{1}^{t} \geq \underline{a}_{1}$, since $D_{2}\left(a_{1}^{t}, r\right) \geq D_{2}\left(\underline{a}_{1}, r\right)>0$, it follows that $\tilde{C}_{2}\left(s^{t}\right) \geq D_{2}\left(\underline{a}_{1}, r\right)$ as in (b) above. In the case where $a_{2}^{t} \geq \underline{a}_{2}, \tilde{C}_{2}\left(s^{t}\right) \geq \underline{a}_{2}$, as in (a) again. So $\tilde{C}_{2}\left(s^{t}\right) \geq \min \left\{D_{2}\left(\underline{a}_{1}, r\right), \underline{a}_{2}\right\}$. Thus taking (a) - (c) together, there is a positive lower bound on $\tilde{C}_{2}\left(s^{t}\right)$; the bound depends on $r$ but we can take its minimum over all $r \in \mathcal{S}$ such that $y(a, r) \geq \mu_{1}$ for some $a \geq 0$; this minimum thus depends only on $V_{1}$ (fixed in the proof). So, in the same way we showed that $c_{1}^{t} \geq \mu_{1}$ for some $t<t^{\prime}$, we can show there exists $t^{\prime \prime} \geq t^{\prime}$ and $\mu_{2}>0$ such that $c_{2}^{\tilde{t}} \geq \mu_{2}$ for some $\tilde{t}<t^{\prime \prime}$ with positive probability. Next, if $\hat{V}_{2} \geq V_{2}$, we can repeat the argument in a symmetric fashion. Denote the minima of $\mu_{1}$ and $\mu_{2}$ over the two cases $\hat{V}_{1} \geq V_{1}$ and $\hat{V}_{2} \geq V_{2}$ by $\mu_{1}$ and $\mu_{2}$, and the maximum of $t^{\prime \prime}$ by $\underline{t}^{\prime \prime}$. So for both agents, $c_{i}^{t} \geq \mu_{i}>0$, for some $t<\underline{t}^{\prime \prime}$ (not necessarily at the same date $t$ for each agent) with probability at least equal to the minimum probability $\underline{\pi}$, say, of any $\underline{t}^{\prime \prime}$-period positive probability history emanating from $s_{0}$ where $\underline{\pi}>0$ as $\mathcal{S}$ is finite. Note that $\mu_{1}, \mu_{2}, \underline{t}^{\prime \prime}$, and $\underline{\pi}$ are all independent of the particular optimum at $t=0$.

(ii) If no $\left(V_{1}, V_{2}\right) \in \Lambda\left(\mathcal{V}_{s}\right)$ exists with $V_{1}, V_{2}>0$, then there exists a unique optimum $\left(V_{1}>0, V_{2}=0\right)$, or a unique optimum $\left(V_{1}=0, V_{2}>0\right)$, or both points exist as optima. In either case the argument above can be repeated mutatis mutandis.

Let $\hat{t}$ (random) denote the earliest date such that both consumptions have been positive, i.e., the first period for which $c_{1}^{t}>0$ and $c_{2}^{\tilde{t}}>0$ for $t, \tilde{t}, \leq \hat{t}$. By optimality, whenever $s_{0}$ occurs on a positive probability history, utilities must belong to $\Lambda\left(\mathcal{V}_{s_{0}}\right)$. From the above argument and given that all states communicate so that $s_{0}$ is recurrent, then we conclude that $\hat{t}$ is finite almost surely. After $\hat{t}$, both $c_{1}$ and $c_{2}$ have been positive at some point in the past. From Theorem 1(ii) we know that for $t>\hat{t}, a_{i}^{t} \leq a_{i}^{*}\left(a_{j}^{t}\right)$, $i=1,2$. Suppose that one agent, say agent $i$, is unconstrained at $t>\hat{t}$. Then from 
Theorem 1(i), $a_{i}^{t} \geq a_{i}^{*}\left(a_{j}^{t}\right), j \neq i$, whilst from Lemma $6(\mathrm{i}), a_{j}^{t} \geq a_{j}^{*}\left(a_{i}^{t}\right)$. Hence if either agent is unconstrained, $a^{t}=a^{* *}$ and actions are efficient. Inefficiency can only occur if both agents are constrained. But from Lemma 8 and Lemma 9 joint utility and current surplus is maximized thereafter. We conclude then that on any path after $\hat{t}$ either actions are always efficient, or there is at most one date at which actions are not efficient, but this is then followed by the current surplus maximizing actions thereafter. Q.E.D.

Proof of Theorem 3: To simplify notation where there is no ambiguity we shall write $a_{i}^{*}\left(a_{j}^{t}\right)$ as $a_{i}^{*}$ and suppress the current state $s_{t}$.

Step 1: We show first that if at $t \geq 0, c_{1}^{t}=0$, we have $a_{1}^{t} \geq a_{1}^{*}$ and $a_{2}^{t} \leq a_{2}^{*}$. Moreover, if $t \geq 1$ and either inequality is strict, then if $c_{1}^{t-1}=0, a_{1}^{t-1}>a_{1}^{*}$. (And likewise if the agent indices are swapped.) To see the first part of the claim, note that by $a_{i}^{t} \geq a_{i}^{N E}>0$, $i=1,2, y^{t}>0$ and so $c_{2}^{t}>0$; thus $a_{2}^{t} \leq a_{2}^{*}$ from Lemma 6(ii). Moreover, by Lemma 7, agent 2 is unconstrained ( $\operatorname{as} a_{2}^{t}>0$ ); hence $a_{1}^{t} \geq a_{1}^{*}$. For the second part of the claim, we shall consider changing, say increasing, agent 1 's utility at $t$ a small amount by decreasing $a_{1}$ and at the same time (as this will have relaxed agent 1's constraint) increasing $a_{2}$ so that agent 1's constraint is unaffected (so if it initially binds, it remains satisfied but binding), while holding $c_{1}=0$, and the future contract fixed. Formally, let $V_{i}^{t}$ denote current (to $t$ ) discounted equilibrium utility and $V_{i, r}^{t+1}$ the same at $t+1$ in state $r$. Then consider the equations

$$
\begin{aligned}
V_{1}+a_{1} & =\delta \sum_{r \in \mathcal{S}} \pi_{s_{t} r} V_{1, r}^{t+1}, \\
V_{2}-y\left(a_{1}, a_{2}\right)+a_{2} & =\delta \sum_{r \in \mathcal{S}} \pi_{s_{t} r} V_{2, r}^{t+1}, \\
V_{1}-D_{1}\left(a_{2}\right) & =V_{1}^{t}-D_{1}\left(a_{2}^{t}\right) .
\end{aligned}
$$

These are satisfied by definition at $\left(V_{1}, V_{2}, a_{1}, a_{2}\right)=\left(V_{1}^{t}, V_{2}^{t}, a_{1}^{t}, a_{2}^{t}\right)$ (noting that the equality $c_{2}=y\left(a_{1}, a_{2}\right)$ has been used to substitute out for $c_{2}$ in the second line). As the functions $y\left(a_{1}, a_{2}\right), D_{1}\left(a_{2}\right)$ and $D_{2}\left(a_{1}\right)$ are continuously differentiable, and $\partial D_{1}\left(a_{2}^{t}\right) / \partial a_{2} \neq 0$ (as $\partial D_{1}\left(a_{2}^{t}\right) / \partial a_{2}>0$ from Lemma 3 , and given $0<a_{2}^{t} \leq a_{2}^{*}$ implies $\left.\partial y\left(a_{1}^{t}, a_{2}^{t}\right) / \partial a_{2}>0\right)$, the implicit function theorem asserts the existence of continuously differentiable functions $a_{1}\left(V_{1}\right), a_{2}\left(V_{1}\right)$ and $V_{2}\left(V_{1}\right)$ in an open interval around $V_{1}^{t}$ such that $a_{1}\left(V_{1}^{t}\right)=a_{1}^{t}$ etc. which satisfy (A.14), and such that

$$
V_{2}^{\prime}\left(V_{1}^{t}\right)=-\frac{\partial y\left(a_{1}^{t}, a_{2}^{t}\right)}{\partial a_{1}}-\frac{\left(1-\frac{\partial y\left(a_{1}^{t}, a_{2}^{t}\right)}{\partial a_{2}}\right)}{\frac{\partial D_{1}\left(a_{2}^{t}\right)}{\partial a_{2}}} .
$$

Agent 2 is unconstrained so remains unconstrained for small changes in $V_{1}$ away from $V_{1}^{t}$, the value at the optimum, while the third line of (A.14) ensures that agent 1's constraint holds. Therefore the continuation contracts from date $t$, defined as $V_{1}$ is varied locally (i.e., with $a$ and $c_{2}$ varying and holding the future fixed), are self-enforcing, and $V_{2}\left(V_{1}\right)$ gives agent 2's utility from each such contract. Now suppose that either inequality is strict, i.e., $a_{1}^{t}>a_{1}^{*}$ or $a_{2}^{t}<a_{2}^{*}$. Then $V_{2}^{\prime}\left(V_{1}^{t}\right)>-1$ (recall $\partial D_{1}\left(a_{2}^{t}\right) / \partial a_{2}>0$ ). At $t-1$, suppose that $a_{1}^{t-1} \leq a_{1}^{*}$. A small increase in $a_{1}^{t-1}$ of $\Delta>0$ holding $c_{1}^{t-1}=0$ (recall $c_{1}^{t-1}=0$ by hypothesis), leads to an increase in agent 2's payoff of at least, to a 
first-order approximation, $\Delta$ (since $\partial y / \partial a_{1} \geq 1$ ) while agent 1 can be compensated at $t$ by a value of $V_{1}$ satisfying $\left(V_{1}-V_{1}^{t}\right)=\Delta / \delta \pi_{s_{t-1} s_{t}}$, so that agent 2's utility changes by approximately $\Delta+\delta \pi_{s_{t-1} s_{t}} V_{2}^{\prime}\left(V_{1}\right)\left(V_{1}-V_{1}^{t}\right)>0$. Agent 1's constraint at $t-1$ holds: her utility is held constant and her deviation payoff is unchanged $\left(a_{2}^{t-1}\right.$ is unchanged); and agent 2's constraint must hold by Lemma $7\left(c_{1}^{t-1}=0\right)$. By construction of the contracts defined for each $V_{1}$ the constraints hold from $t$. Thus a Pareto-improvement has been found for $\Delta$ small enough, contrary to the assumed optimality. Hence $a_{1}^{t-1}>a_{1}^{*}$.

Step 2: Suppose at a positive probability $s^{t^{\prime}}, t^{\prime} \geq 0$, that $a_{1}^{t^{\prime}}>a_{1}^{*}$. Then we have $c_{1}^{t}=0$ for all $t \leq t^{\prime}$ (by Lemma 6(ii) and Theorem 1(ii)). However, $a_{1}^{t^{\prime}}>a_{1}^{*}$ then implies by repeated application of Step 1 that $a_{1}^{t}>a_{1}^{*}$ for all $t<t^{\prime}$. Moreover as $c_{2}^{t}>0$ for all $t \leq t^{\prime}, a_{2}^{t} \leq a_{2}^{*}$ for all $t \leq t^{\prime}$. Hence Phase 1 conditions are satisfied for all $t \leq t^{\prime}$ and for $i=1$. By the same logic, if at any point $t^{\prime}$ the Phase 2 conditions $a^{t^{\prime}} \leq a^{*}$ hold, they must hold at all subsequent positive probability histories, since a violation (i.e., $a_{i}^{t}>a_{i}^{*}$ for $t>t^{\prime}$ and some $i$ ) would imply that $a_{i}^{t^{\prime}}>a_{i}^{*}$ also. Thus any positive probability $s^{t^{\prime}}$ must satisfy the two-phase property up to $t^{\prime}$. The fact that $\tilde{t}$ in the statement of the theorem is a.s. finite follows from the argument in the proof of convergence that the date at which both consumptions have been positive is itself a.s. finite (as both consumptions positive implies $a^{t} \leq a^{*}$ by Theorem 1(ii)).

Step 3: Next, we consider Phase 2, after the initial period $\tilde{t}$. Suppose that at $\tilde{t}+1$ in some state $r$ with $\pi_{\tilde{s}^{r}}>0$ at least one constraint does not bind. Suppose w.l.o.g. that agent 2 is unconstrained and we can repeat the argument of Step 1, with $c_{1}$ again being held constant, but now at a possibly positive level. Again we have a locally differentiable relationship between utilities arising from self-enforcing contracts at $\tilde{t}+1$, with slope $V_{2}^{\prime}\left(V_{1}^{\tilde{t}+1}\right)$ given by (A.15). As agent 2 is unconstrained $a_{1}^{\tilde{t}+1} \geq a_{1}^{*}$, and by Phase $2, a_{1}^{\tilde{t}+1} \leq a_{1}^{*}$, so $a_{1}^{\tilde{t}+1}=a_{1}^{*}$. Consequently if $a_{2}^{\tilde{t}+1}<a_{2}^{*}$, then $V_{2}^{\prime}\left(V_{1}^{\tilde{t}+1}\right)>-1$, and repeating the argument at the end of Step 1, $a_{1}^{\tilde{t}}>a_{1}^{*}$, which contradicts the definition of $\tilde{t}$. So $a_{2}^{\tilde{t}+1}=a_{2}^{*}$. Thus it must be (if either agent is unconstrained at $\tilde{t}+1$ ) that $a^{\tilde{t}+1}=a^{* *}$. It follows that if $a^{\tilde{t}+1} \neq a^{* *}$, both agents are constrained, and it cannot be that $c_{i}^{\tilde{t}+1}=0$ for either agent as that would imply agent $j \neq i$ is unconstrained by Lemma 7 (as $\left.a_{j}^{\tilde{t}+1}>0\right)$. Hence the only alternative is that both are constrained, and $c_{i}^{\tilde{t}+1}>0, i=1,2$.

Step 4: Moreover if this latter is the case $\left(a^{\tilde{t}+1} \neq a^{* *}\right.$, with both being constrained, and $\left.c_{i}^{\tilde{t}+1}>0, i=1,2\right)$, it cannot be that $a_{i}^{\tilde{t}+1}=a_{i}^{*}$ for either $i=1$ or 2 . Suppose to the contrary and that w.l.o.g. $a_{1}^{\tilde{t}+1}=a_{1}^{*}$. Again we can construct a family of self-enforcing contracts by varying the contract only at $\tilde{t}+1$. Consider the equations

$$
\begin{aligned}
V_{1}-c_{1}+a_{1} & =\delta \sum_{r \in \mathcal{S}} \pi_{s r} V_{1, r}^{\tilde{t}+2}, \\
V_{2}-y\left(a_{1}, a_{2}\right)+c_{1}+a_{2} & =\delta \sum_{r \in \mathcal{S}} \pi_{s r} V_{2, r}^{\tilde{t}+2} \\
V_{1}-D_{1}\left(a_{2}\right) & =0 \\
V_{2}-D_{2}\left(a_{1}\right) & =0
\end{aligned}
$$

where $V_{i, r}^{\tilde{t}+2}$ is continuation utility for $i$ on the equilibrium path from $\tilde{t}+2$ in state $r$. These are satisfied at $\left(V_{1}^{\tilde{t}+1}, V_{2}^{\tilde{t}+1}, c_{1}^{\tilde{t}+1}, a_{1}^{\tilde{t}+1}, a_{2}^{\tilde{t}+1}\right)$. As the functions $y\left(a_{1}, a_{2}\right), D_{1}\left(a_{2}\right)$ and 
$D_{2}\left(a_{1}\right)$ are continuously differentiable, the implicit function theorem asserts, provided that

$$
\frac{\partial D_{1}\left(a_{2}^{\tilde{t}+1}\right)}{\partial a_{2}}\left(1+\frac{\partial D_{2}\left(a_{1}^{\tilde{t}+1}\right)}{\partial a_{1}}-\frac{\partial y\left(a_{1}^{\tilde{t}+1}, a_{2}^{\tilde{t}+1}\right)}{\partial a_{1}}\right) \neq 0,
$$

the existence of continuously differentiable functions $c_{1}\left(V_{1}\right), a_{1}\left(V_{1}\right), a_{2}\left(V_{1}\right)$ and $V_{2}\left(V_{1}\right)$ in an open interval around $V_{1}^{\tilde{t}+1}$ such that $c_{1}\left(V_{1}^{\tilde{t}+1}\right)=c_{1}^{\tilde{t}+1}$ etc. which satisfy equation (A.16). As $a_{1}^{\tilde{t}+1}=a_{1}^{*}, \partial y\left(a_{1}^{\tilde{t}+1}, a_{2}^{\tilde{t}+1}\right) / \partial a_{1}=1$, and as $\partial D_{i}\left(a_{j}^{\tilde{t}+1}\right) / \partial a_{j}>0$ (from Lemma 3, given that $\partial y\left(a_{i}^{N}\left(a_{j}^{\tilde{t}+1}\right), a_{j}^{\tilde{t}+1}\right) / \partial a_{j}>0$ by monotonicity of $y(\cdot)$ and $a_{i}^{N}\left(a_{j}^{\tilde{t}+1}\right)>0$, the latter following from $a_{j}^{\tilde{t}+1} \geq a_{j}^{N E}>0$ and the nondecreasing reaction function) it follows that (A.17) holds. Since $c_{1}^{\tilde{t}+1}>0$ and $c_{2}^{\tilde{t}+1}>0$, nonnegativity constraints on consumption will also hold in an open interval around $V_{1}^{\tilde{t}+1}$, and the self-enforcing constraints hold (by the third and fourth lines of (A.16)). Hence holding the future contract fixed, but varying $V_{1}$, varies the current contract according to $c_{1}\left(V_{1}\right)$, $a_{1}\left(V_{1}\right), a_{2}\left(V_{1}\right)$, and traces out a series of self-enforcing contracts such that agent 2's discounted utility is $V_{2}\left(V_{1}\right)$, with

$$
V_{2}^{\prime}\left(V_{1}^{\tilde{t}+1}\right)=-\frac{\frac{\partial D_{2}\left(a_{1}^{\tilde{t}+1}\right)}{\partial a_{1}}\left(1+\frac{\partial D_{1}\left(a_{2}^{\tilde{t}+1}\right)}{\partial a_{2}}-\frac{\partial y\left(a_{1}^{\tilde{t}+1}, a_{2}^{\tilde{t}+1}\right)}{\partial a_{2}}\right)}{\frac{\partial D_{1}\left(a_{2}^{\tilde{t}+1}\right)}{\partial a_{2}}\left(1+\frac{\partial D_{2}\left(a_{1}^{\tilde{t}+1}\right)}{\partial a_{1}}-\frac{\partial y\left(a_{1}^{\tilde{t}+1}, a_{2}^{\tilde{t}+1}\right)}{\partial a_{1}}\right)} .
$$

As $a_{2}^{\tilde{t}+1}<a_{2}^{*}, \partial y\left(a_{1}^{\tilde{t}+1}, a_{2}^{\tilde{t}+1}\right) / \partial a_{2}>1$, so given $\partial y\left(a_{1}^{\tilde{t}+1}, a_{2}^{\tilde{t}+1}\right) / \partial a_{1}=1$ it follows that $V_{2}^{\prime}\left(V_{1}^{\tilde{t}+1}\right)>-1$, which we have shown is impossible as again it would imply $a_{1}^{\tilde{t}}>a_{1}^{*}$. Hence a contradiction, and therefore if both agents are constrained it is concluded that $a_{i}^{\tilde{t}+1}<a_{i}^{*} \leq a_{i}^{* *}, i=1,2$.

Q.E.D.

Proof of Theorem 4: Suppose to the contrary that $a_{1}^{t}>a_{1}^{*}$ for some $s^{t}$. From Lemma 6(ii), $c_{1}^{t}=0$. Agent 1's optimal current payoff from defaulting is just the Nash breakdown payoff: $\phi_{1}\left(a_{1}^{N E}, s_{t}\right)-a_{1}^{N E}$. We thus have equilibrium current utility, $w_{1}^{t}$, is less than this breakdown payoff, as $a_{1}^{N E} \leq a_{1}^{*}<a_{1}^{t}$ and $c_{1}^{t}=0 \leq \phi_{1}\left(a_{1}^{N E}, s_{t}\right)$. Denote this negative surplus by $\chi^{t} \equiv w_{1}^{t}-\left(\phi_{1}\left(a_{1}^{N E}, s_{t}\right)-a_{1}^{N E}\right)<0$. Agent 1 's discounted utility is $V_{1}\left(s^{t}\right)=w_{1}^{t}+\delta \sum_{s_{t+1} \in \mathcal{S}} \pi_{s_{t} s_{t+1}} V_{1}\left(s^{t+1}\right)$, so defining the discounted surplus as $V S_{1}\left(s^{t}\right) \equiv V_{1}\left(s^{t}\right)-D_{1}\left(s_{t}\right)$ we have

$$
V S_{1}\left(s^{t}\right)=\chi\left(s^{t}\right)+\delta \sum_{s_{t+1} \in \mathcal{S}} \pi_{s_{t} s_{t+1}} V S_{1}\left(s^{t+1}\right) \geq 0 .
$$

From equation (A.18) it follows that there is at least one state at date $t+1$ with $\pi_{s_{t} s_{t+1}}>0$ such that $V S_{1}\left(s^{t+1}\right) \geq-\chi\left(s^{t}\right) / \delta>0$. Suppose that either $a_{1}^{t+1}=0$ or $a_{1}^{t+1}>a_{1}^{*}$. In the former case, $f\left(a_{1}^{t+1}, s_{t+1}\right)=0$, so $w_{1}^{t+1}=0$. In the latter case, from Lemma 6 (ii), $c_{1}^{t+1}=0$, so $w_{1}^{t+1}<0$. Consequently, there must, by repeating the earlier logic, be another successor state at date $t+2$ with $\pi_{s_{t+1} s_{t+2}}>0$ such that continuation surplus $V S_{1}\left(s^{t+2}\right) \geq-\chi\left(s^{t}\right) / \delta^{2}$. We can repeat this argument if again either $a_{1}^{t+2}=0$ or $a_{1}^{t=2}>a_{1}^{*}$. Since continuation surplus must be bounded, this can only happen a fixed number of times. Thus we must have (along such a 
path) in finite time $t^{\prime}>t, 0<a_{1}^{t^{\prime}} \leq a_{1}^{*}\left(s_{t^{\prime}}\right)$ and $V S_{1}\left(s^{t^{\prime}}\right)>0$ for the first time. Suppose first this happens at $t^{\prime}=t+1$. Thus in this state at $t+1$, agent 1 is unconstrained. Consider frontloading agent 1's utility by increasing her action at $t+1$ in state $s_{t+1}$ by $\Delta>0$ and reducing it by $\delta \pi_{s_{t} s_{t+1}} \Delta$ at date $t$ to compensate (holding consumption constant). Agent 2's utility changes (to a first-order approximation) by $-\left(\partial f\left(a_{1}^{t}, s_{t}\right) / \partial a_{1}\right) \delta \pi_{s_{t} s_{t+1}} \Delta+\delta \pi_{s_{t} s_{t+1}}\left(\partial f\left(a_{1}^{t+1}, s_{t+1}\right) / \partial a_{1}\right) \Delta$ which is positive by virtue of $a_{1}^{t}>a_{1}^{*}\left(\right.$ so $\left.\partial f\left(a_{1}^{t}, s_{t}\right) / \partial a_{1}<1\right)$ and $0<a_{1}^{t+1} \leq a_{1}^{*}\left(s_{t+1}\right)\left(\right.$ so $\left.\partial f\left(a_{1}^{t+1}, s_{t+1}\right) / \partial a_{1} \geq 1\right)$. No constraints are violated by this: agent 1 is unconstrained at $t+1\left(V S_{1}\left(s^{t+1}\right)>0\right)$ so for $\Delta$ small her constraint is maintained; agent 2 receives the extra output at $t+1$ and by Assumption 3 his breakdown payoff increases by at most this amount, so his constraint holds. At $t$ there is a Pareto-improvement and agent 2's breakdown payoff has not increased (and agent 1's is constant) so again the constraints hold. Thus we have a contradiction. The remaining possibility is that $t^{\prime}>t+1$. A similar construction will lead to a Pareto-improvement at $t$, but now we have additionally to worry about constraints for periods $\hat{t}$ between $t$ and $t+1$. By construction $V S_{1}\left(s^{\hat{t}}\right)>0$ along the entire path, so for $\Delta$ small enough agent 1's continuation surplus remains positive. Agent 2's utility is backloaded, so his constraints are relaxed. Again we have a contradiction. Q.E.D.

Proof of Theorem 5: As usual we drop time superscripts etc. where no ambiguity arises. First, consider any self-enforcing contract in the model without savings, that is (using the shorthand notation $y^{t}=y\left(a\left(s^{t}\right), s_{t}\right)$, etc.), where $c_{i}^{t}=\chi_{i}^{t} \in\left[0, y^{t}\right]$ for all $t \geq 0$. Since the contract is self-enforcing $V_{i}^{t} \geq D_{i}\left(a_{j}^{t}\right)$. This contract is self-enforcing when savings are possible as it specifies that transfers are consumed immediately; that is, $V_{i}^{t} \geq D_{i}\left(a_{j}^{t}\right)+S_{i}^{t}$ since in this putative equilibrium $S_{i}^{t}=0$, and so the deviation payoffs are unchanged (agents can do no better than consume current transfers when they deviate).

To establish the converse, it is sufficient to start from a Pareto-efficient equilibrium in the model with savings (existence follows along the lines of the proof of Lemma 4). We show that the same ex ante utilities can be generated in a self-enforcing contract without savings. The proof proceeds in four stages. First we show that it is feasible to change transfers over the first $T$ periods of the contract to satisfy the three properties outlined in the text. Second we show that there exists a limit contract which satisfies these properties. Thirdly we modify the changed contract to eliminate some potential overinvestment. Lastly we show that this new contract satisfies the self-enforcing constraints.

Step 1: In general for any efficient self-enforcing contract with savings we adopt a similar procedure to the example in the text and working backwards from some date $T \geq 1$ make a sequence of transfer changes $\Delta \chi_{i}\left(s^{t}, T\right)$ at each $s^{t}, t \leq T$, where $T$ is the last date at which changes are to be made $\left(\Delta \chi_{i}\left(s^{t}, T\right)=0\right.$ for $\left.t>T\right)$. Keep actions fixed and define the expected discounted change in transfers for agent $i$ from $t$ to be

$$
\theta_{i}\left(s^{t}, T\right)=\Delta \chi_{i}\left(s^{t}, T\right)+\mathrm{E}\left[\sum_{\tau=t+1}^{T} \delta^{\tau-t} \Delta \chi_{i}\left(s^{\tau}, T\right) \mid s^{t}\right] \quad \text { for } t \leq T .
$$

Since these are transfers between the two agents we have $\theta_{i}\left(s^{t}, T\right)=-\theta_{j}\left(s^{t}, T\right), i \neq j$. At each date $t \leq T$ the changes are made to make transfers non-negative, and to the maximum extent possible, to compensate for the subsequent changes along successor 
histories. In other words, given the changes which have been made from $t+1$ until $T$, the problem at $t \geq 1$ is to choose $\Delta \chi_{i}\left(s^{t}, T\right)$ to minimize $\left|\theta_{i}\left(s^{t}, T\right)\right|$ subject to $0 \leq \chi_{i}\left(s^{t}\right)+\Delta \chi_{i}\left(s^{t}, T\right) \leq y\left(a\left(s^{t}\right), s_{t}\right)$. For each $T$ and history $s^{t}, t \leq T$, this process satisfies the three properties identified in the text: the new transfers are non-negative, if the discounted transfers of an agent falls $\left(\theta_{i}\left(s^{t}, T\right)<0\right)$ then she is allocated all of the current output (correspondingly $\theta_{i}\left(s^{t}, T\right)>0$ implies $\chi_{i}\left(s^{t}\right)+\Delta \chi_{i}\left(s^{t}, T\right)=0$ ), and we shall now show that the initial discounted transfer distribution is unchanged.

As the largest possible transfer is constrained by the inherited savings, $-S_{i}^{T} \leq$ $\chi_{i}\left(s^{T}\right) \leq y^{T}+S_{j}^{T}$, and as savings are non-negative we have for each $s^{T}$ that $S_{i}^{T} \geq$ $\max \left\{0, \Delta \chi_{i}\left(s^{T}, T\right)\right\}, i=1,2$. This says that since the original allocation was feasible whichever agent is called upon to make the transfer had sufficient savings to make that transfer. From $\theta_{i}\left(s^{T}, T\right) \equiv \Delta \chi_{i}\left(s^{T}, T\right)$, we get $-S_{j}^{T} \leq \theta_{i}\left(s^{T}, T\right) \leq S_{i}^{T}$.

Next suppose that at $t+1,-S_{j}^{t+1} \leq \theta_{i}\left(s^{t+1}, T\right) \leq S_{i}^{t+1}$, all $s^{t+1}$. We shall establish the induction step that $-S_{j}^{t} \leq \theta_{i}\left(s^{t}, T\right) \leq S_{i}^{t}$ for all $s^{t}$. When $\theta_{i}\left(s^{t}, T\right)=-\theta_{j}\left(s^{t}, T\right)=0$ it follows trivially. Thus suppose that at $s^{t}$ (considered fixed for the following argument) $\theta_{i}\left(s^{t}, T\right)>0$. There are two cases. (a) $\theta_{i}\left(s^{t}, T\right)+\chi_{i}\left(s^{t}\right)<0$. Then by feasibility $S_{i}^{t} \geq-\chi_{i}\left(s^{t}\right)>\theta_{i}\left(s^{t}, T\right)$. (b) $\theta_{i}\left(s^{t}, T\right)+\chi_{i}\left(s^{t}\right) \geq 0$. Define $\bar{\theta}_{i}\left(s^{t+1}, T\right):=$ $\max _{s_{t+1}} \theta_{i}\left(\left(s^{t}, s_{t+1}\right), T\right)$. We have

$$
\begin{aligned}
S_{i}^{t} \geq(1+r)^{-1} S_{i}^{t+1}-\chi_{i}\left(s^{t}\right) & \geq(1+r)^{-1} \bar{\theta}_{i}\left(s^{t+1}, T\right)-\chi_{i}\left(s^{t}\right) \\
& \geq(1+r)^{-1} \delta^{-1}\left(\theta_{i}\left(s^{t}, T\right)-\Delta \chi_{i}\left(s^{t}, T\right)\right)-\chi_{i}\left(s^{t}\right) \\
& =(1+r)^{-1} \delta^{-1}\left(\theta_{i}\left(s^{t}, T\right)+\chi_{i}\left(s^{t}\right)\right)-\chi_{i}\left(s^{t}\right) \\
& \geq \theta_{i}\left(s^{t}, T\right) .
\end{aligned}
$$

The first inequality follows as $S_{i}^{t+1} \leq(1+r)\left(\chi_{i}\left(s^{t}\right)+S_{i}^{t}\right)$, the second from the induction assumption $S_{i}^{t+1} \geq \bar{\theta}_{i}\left(s^{t+1}, T\right)$, the third from $\Delta \chi_{i}\left(s^{t}, T\right) \geq \theta_{i}\left(s^{t}, T\right)-\delta \bar{\theta}_{i}\left(s^{t+1}, T\right)$ (which follows from (A.19) and the definition of $\bar{\theta}_{i}\left(s^{t+1}, T\right)$ ), the equality from $\chi_{i}\left(s^{t}\right)+$ $\Delta \chi_{i}\left(s^{t}, T\right)=0$ (recall $\theta_{i}\left(s^{t}, T\right)>0$ implies a modified transfer of zero), and the last inequality as $\delta(1+r) \leq 1$ and $\theta_{i}\left(s^{t}, T\right)+\chi_{i}\left(s^{t}\right) \geq 0$.

We have established that $\theta_{i}\left(s^{t}, T\right) \leq S_{i}^{t}$ whenever $\theta_{i}\left(s^{t}, T\right)>0$, and so $-S_{j}^{t} \leq$ $\theta_{i}\left(s^{t}, T\right) \leq S_{i}^{t}$. It follows that $S_{j}^{t} \geq-\theta_{i}\left(s^{t}, T\right)=\theta_{j}\left(s^{t}, T\right) \geq-S_{i}^{t}$, so the bounds apply also for $\theta_{j}\left(s^{t}, T\right)<0$. This completes the induction argument, and at date 0 , from $S_{1}^{0}=S_{2}^{0}=0$, we therefore have $\theta_{i}\left(s^{t}, T\right)=0, i=1,2$. That is, the initial discounted transfer distribution is unchanged, as was to be shown.

Step 2: We know the action space is bounded (see Lemma 4), so output is also bounded. Then there is some number $B$ such that the sum of current output (at $t$ ) and total continuation utility from date $t+1$ on discounted to date $t$, is bounded above. Hence, denoting utility from the end of $t$ (i.e., gross of action costs) by $\widetilde{V}_{i}^{t}$, we have $\widetilde{V}_{1}^{t}+\widetilde{V}_{2}^{t} \leq$ $B+S_{1}^{t}+S_{2}^{t}$. We also have $\widetilde{V}_{i}^{t} \geq S_{i}^{t}$ as agent $i$ can guarantee a utility at least equal to inherited resources. Then if the equilibrium transfer from $i$ to $j$ is $B+\epsilon$ for $\epsilon>0$, we must have $\widetilde{V}_{j}^{t} \geq B+\epsilon+S_{j}^{t}$ since $j$ could just consume the current transfer and inherited resources. But then we have $\widetilde{V}_{i}^{t} \leq S_{i}^{t}-\epsilon<S_{i}^{t}$ which is impossible. Thus transfers are bounded by $B$.

For each $T$ we have defined $\Delta \chi_{i}\left(s^{t}, T\right)$ for all $s^{t}$ (with $\Delta \chi_{i}\left(s^{t}, T\right)=0$ for $t>T$ ). Consider now the sequence of the changed transfers as $T \rightarrow \infty$; given that actions 
and transfers are bounded, we can use sequential compactness in the product topology (along the lines of Lemma 4) to guarantee the existence of a convergent subsequence $T^{(p)}$ for $p=1,2, \ldots$. The limit of this subsequence then gives us our changed contract. That is we denote $\Delta \chi_{i}\left(s^{t}\right):=\lim _{p \rightarrow \infty} \Delta \chi_{i}\left(s^{t}, T^{(p)}\right)$ and define the changed contract as $\tilde{\chi}_{i}\left(s^{t}\right):=\chi_{i}\left(s^{t}\right)+\Delta \chi_{i}\left(s^{t}\right)$. The limit changes satisfy the same properties as above at all dates. Since the transfers converge to some limit so too does the discounted value of the expected changes $\theta_{i}\left(s^{t}, T\right)$ (as it is a continuous function of these changes), and write $\theta_{i}\left(s^{t}\right):=\lim _{p \rightarrow \infty} \theta_{i}\left(s^{t}, T^{(p)}\right)$. This limit too satisfies the above property that it is positive only when the new transfer is zero and is negative only when the agent receives all the output. Finally, we specify that there is no saving, so that all transfers are consumed immediately.

Step 3: Possibly at $s^{t}$ the modified contract has $\tilde{\chi}_{i}\left(s^{t}\right)=y\left(a^{t}\left(s^{t}\right), s_{t}\right)$ and $a_{i}\left(s^{t}\right)>$ $a_{i}^{*}\left(a_{j}\left(s^{t}\right), s_{t}\right)$. Nevertheless it is feasible to reduce $a_{i}\left(s^{t}\right)$ to $a_{i}^{*}\left(a_{j}\left(s^{t}\right), s_{t}\right)$ and simultaneously reduce $\tilde{\chi}_{i}\left(s^{t}\right)$ by the consequent change in output. That is, we change the contract (again) for all such histories so the new actions and transfers satisfy $\hat{a}_{i}\left(s^{t}\right)=a_{i}^{*}\left(a_{j}\left(s^{t}\right), s_{t}\right)$ and $\hat{\chi}_{i}\left(s^{t}\right)=y\left(a_{i}^{*}\left(a_{j}\left(s^{t}\right), s_{t}\right), a_{j}\left(s^{t}\right), s_{t}\right)$. This raises the utility of agent $i$ as inefficiency has been reduced (and the incentive constraint of agent $j$ is relaxed because $a_{i}$ has fallen). After having made these changes denote the changed continuation utilities by $\hat{U}_{i}\left(s^{t}\right)$ and $\hat{V}_{i}\left(s^{t}\right)$.

Step 4: First consider $s^{t}$ such that $\theta_{i}\left(s^{t}\right) \geq 0$. Also in the original contract $V_{i}\left(s^{t}\right) \leq$ $U_{i}\left(s^{t}\right)+S_{i}^{t}$. Since after step 3 the contract satisfies $\hat{U}_{i}\left(s^{t}\right) \geq U_{i}\left(s^{t}\right)+\theta_{i}\left(s^{t}\right)$ (the inequality is strict if actions have been reduced because of overinvestment as indicated in step 3 ) and the new contract has no saving,

$$
\hat{V}_{i}\left(s^{t}\right)=\hat{U}_{i}\left(s^{t}\right) \geq U_{i}\left(s^{t}\right)+\theta_{i}\left(s^{t}\right) \geq V_{i}\left(s^{t}\right)-S_{i}^{t} .
$$

It then follows from the original default constraint (4) that $\hat{V}_{i}\left(s^{t}\right) \geq D_{i}\left(a_{j}\left(s^{t}\right)\right) \geq$ $D_{i}\left(\hat{a}_{j}\left(s^{t}\right)\right)$, where the final inequality follows since $a_{j}\left(s^{t}\right) \geq \hat{a}_{j}\left(s^{t}\right)$ by the changes made to actions above. Hence the incentive constraint is satisfied.

Next consider the case where $\theta_{i}\left(s^{t}\right)<0$ and hence $\hat{\chi}_{i}\left(s^{t}\right)=y\left(\hat{a}_{i}\left(s^{t}\right), a_{j}\left(s^{t}\right)\right)$. (Since we know $\theta_{j}\left(s^{t}\right)>0$ and hence $\tilde{\chi}_{j}\left(s^{t}\right)=0$ we know that the action of agent $j$ is unchanged.) We have (dropping the date and state notation) that $a_{i} \geq a_{i}^{N}\left(a_{j}\right)$ from Lemma 5 as this result applies independently of any savings. Now apply the logic of Lemma 7 to show that the agent's incentive constraint holds. First, $\hat{\chi}_{i}-\hat{a}_{i} \geq \phi_{i}\left(a_{i}^{N}\left(a_{j}\right), a_{j}\right)-a_{i}^{N}\left(a_{j}\right)$, so there is no short-run gain to deviating (this follows as $a_{i}^{N}\left(a_{j}\right) \leq \hat{a}_{i} \leq a_{i}^{*}\left(a_{j}\right)$ and therefore $y\left(\hat{a}_{i}, a_{j}\right)-\hat{a}_{i} \geq y\left(a_{i}^{N}\left(a_{j}\right), a_{j}\right)-a_{i}^{N}\left(a_{j}\right)$, and from $\left.y\left(a_{i}^{N}\left(a_{j}\right), a_{j}\right) \geq \phi_{i}\left(a_{i}^{N}\left(a_{j}\right), a_{j}\right)\right)$. We also need to show that for all $s_{t+1}, \widehat{V}_{i}\left(s^{t}, s_{t+1}\right) \geq D_{i}\left(a_{j}^{N E}\right)$. First we note that if $\theta_{i}\left(s^{\tau}\right)<0$ then the same argument applies and (dropping $s^{\tau}$ etc.) we have $\hat{\chi}_{i}-\hat{a}_{i} \geq$ $\phi_{i}\left(a_{i}^{N}\left(a_{j}\right), a_{j}\right)-a_{i}^{N}\left(a_{j}\right) \geq \phi_{i}\left(a^{N E}\right)-a_{i}^{N E}$ since $\hat{a}_{i} \geq a_{i}^{N E}\left(a_{i} \geq a_{i}^{N E}\right.$ from Lemma 5 again, and if $\hat{a}_{i}<a_{i}, \hat{a}_{i}=a_{i}^{*}\left(a_{j}\right) \geq a_{i}^{N E}\left(a_{j}\right) \geq a_{i}^{N E}\left(a_{j}^{N E}\right)=a_{i}^{N E}$ from Lemma 2 and $a_{i}^{N E}(\cdot)$ non-decreasing) and $\phi_{i}$ non-decreasing. Secondly, if $\theta_{i}\left(s^{\tau}\right) \geq 0$ then the argument in the previous paragraph gives $\hat{V}_{i}\left(s^{\tau}\right) \geq D_{i}\left(a_{j}\left(s^{\tau}\right)\right) \geq D_{i}\left(a_{j}^{N E}\right)$ as $a_{j}\left(s^{\tau}\right) \geq a_{j}^{N E}$. Thus the continuation utility is no less than the breakdown equilibrium along all future paths and hence $\widehat{V}_{i}\left(s^{t}, s_{t+1}\right) \geq D_{i}\left(a_{j}^{N E}\right)$ as required. Thus Lemma 7 applies and the incentive constraint of the agent who gets all the output holds. 
To conclude, we have constructed a self-enforcing contract which offers each agent at least as much ex ante utility as the original contract and there are no savings. It satisfies the incentive constraints in the model without savings and thus is self-enforcing in that model.

Q.E.D.

\section{REFERENCES}

Acemoglu, D., M. Golosov, And A. Tsyvinski (2008): "Political Economy of Mechanisms," Econometrica, 76, 619-641.

Aguiar, M. AND M. Amador (2009): "Growth in the Shadow of Expropriation," Mimeo, Stanford University.

Aguiar, M., M. Amador, and G. Gopinath (2009): "Investment Cycles and Sovereign Debt Overhang," Review of Economic Studies, 76, 1-31.

Albuquerque, R. and H. A. Hopenhayn (2004): "Optimal Lending Contracts and Firm Dynamics," Review of Economic Studies, 71, 285-315.

AtKeson, A. And H. Cole (2008): "A Dynamic Theory of Optimal Capital Structure and Executive Compensation," Mimeo, University of California at Los Angeles.

Baker, G., R. Gibbons, And K. Murphy (2002): "Relational Contracts and the Theory of the Firm," Quarterly Journal of Economics, 117, 39-84.

BoArd, S. (2008): "Relational Contracts and the Value of Loyalty," Mimeo, University of California at Los Angeles.

Bond, E. W. (2003): "Consumption Smoothing and the Time Profile of Self-Enforcing Agreements," Mimeo, Vanderbilt University.

Cooley, T., R. Marimon, and V. Quadrini (2004): "Aggregate Consequences of Limited Contract Enforceability," Journal of Political Economy, 112, 817-847.

Doornik, K. (2006): "Relational Contracting in Partnerships," Journal of Economics \&s Management Strategy, 15, 517-548.

FonG, Y.-F. AND J. LI (2009): "Relational Contracts, Limited Liability, and Employment Dynamics," Mimeo, Kellogg School of Management, Northwestern University.

Garvey, G. (1995): "Why Reputation Favors Joint Ventures over Vertical and Horizontal Integration: A Simple Model," Journal of Economic Behaviour and Organization, 28, 387397.

Halonen, M. (2002): "Reputation and the Allocation of Ownership," Economic Journal, 112, $539-558$.

Hauser, C. And G. Uysal (2006): "On Risk Sharing and Public Goods in Dynamic Contracts," Mimeo, University of Rochester.

Hopenhayn, H. And I. Werning (2008): "Equilibrium Default," Mimeo, University of California at Los Angeles.

Itskнокі, O. (2007): "How Expensive is Commitment?" Mimeo, Harvard University.

Kocherlakota, N. R. (1996): "Implications of Efficient Risk Sharing without Commitment," Review of Economic Studies, 63, 595-610.

Kovrijnykh, N. (2009): "Debt Contracts with Partial Commitment," Mimeo, Arizona State University.

Levin, J. (2003): "Relational Incentive Contracts," American Economic Review, 93, 837-857.

Ligon, E., J. P. Thomas, And T. Worrall (2002): "Informal Insurance Arrangements with Limited Commitment: Theory and Evidence from Village Economies," Review of Economic Studies, 69, 209-244.

Macleod, W. B. And J. Malcomson (1989): "Implicit Contracts, Incentive Compatibility and Involuntary Unemployment," Econometrica, 57, 447-480.

Opp, M. (2010): "Expropriation Risk and Technology: A Theory of Endogenous Property Rights," Mimeo, University of California at Berkeley.

ÖzGür, O. (2004): "A Model of Dynamic Liquidity Contracts," Mimeo, New York University. 
Pavoni, N. (2009): "Optimal Unemployment Insurance with Human Capital Depreciation and Duration Dependence," International Economic Review, 50, 323-362.

Popov, L. (2008): "Enforcement Frictions and Optimal Lending Contracts," Mimeo, Iowa State University.

Quintin, E. (2008): "Contract Enforcement and the Size of the Informal Economy," Economic Theory, 37, 395-416.

RAY, D. (2002): "The Time Structure of Self-Enforcing Agreements," Econometrica, 70, 547-582.

RAYO, L. (2007): "Relational Incentives and Moral Hazard in Teams," Review of Economic Studies, 74, 937-963.

Sigouin, C. (2003): "Investment Decisions, Financial Flows, and Self-Enforcing Contracts," International Economic Review, 44, 1359-1382.

Thomas, J. P. and T. Worrall (1988): "Self-Enforcing Wage Contracts," Review of Economic Studies, 55, 541-554.

(1994): "Foreign Direct Investment and the Risk of Expropriation," Review of Economic Studies, 61, 81-108.

YANG, H. (2009): "Nonstationary Relational Contracts," Mimeo, Ohio State University. 